\title{
Two-dimensional wave radiation and diffraction problems in a flat or sloping seabed environment
}

\author{
Aichun Feng ${ }^{\mathrm{a}}$, Wei Bai ${ }^{\mathrm{b}}$, W. G. Price ${ }^{\mathrm{c}, \mathrm{d}}$ \\ ${ }^{a}$ Department of Civil and Environmental Engineering, National University of Singapore, \\ 117576, Singapore \\ ${ }^{b}$ School of Computing, Mathematics and Digital Technology, Manchester Metropolitan \\ University, Chester Street, Manchester M1 5GD,UK \\ ${ }^{c}$ Fluid Structure Interactions Research Group, University of Southampton, SO17 1BJ, UK \\ ${ }^{d}$ WUT-UoS High Performance Ship Technology Joint Centre, Wuhan, 430063, China
}

\begin{abstract}
Two-dimensional water wave problems are investigated in an environment with a flat or sloping rigid seabed adopting a continuous Rankine source method. All the fluid domain surfaces, that is the free, body and seabed surfaces, are discretized using continuous panels. These panels are positioned exactly on the fluid boundary surfaces and no desingularization technique is required. A new seabed source panel distribution method is developed to accommodate both symmetric and asymmetric seabed profiles. To validate the numerical model comparisons are made with published findings from other mathematical models and experimental data. The presence of a sloped seabed alters the symmetry of the fluid domain, causing wave reflection and shoaling, and therefore, significantly affects the hydrodynamic characteristics of water wave problems. The influence of these topographies on the responses in all three degrees of freedom (heave, sway and roll) of a rigid floating body are investigated and discussed accounting for wave radiation and diffraction problems.
\end{abstract}

Keywords: Flat or sloping seabed, Radiation problem and diffraction problem, Continuous Rankine source

\section{Introduction}

As the water depth decreases in near shore or coastal areas, the influence of the seabed on the hydrodynamic performance, and hence operation, of a floating body increases. The evaluation and design of ships and offshore structures in littoral waters are of particular importance in offshore engineering (Sawaragi, 1995; Journée and Massie, 2001). For example, floating breakwaters in coastal areas (Williams et al., 2000), FPSO and LNG vessels during loading and unloading operations, to name but a few.

*Corresponding Author: Aichun Feng; Email, a.feng@nus.edu.sg 
Yu and Ursell (1961) incorporated the seabed effect into the hydrodynamic analysis of a floating body by assuming the seabed flat. Kim (1969) developed a multipole expansion method to examine the behaviour of a cylinder excited in an oscillatory motion. Both these investigations demonstrated the importance of the seabed on the hydrodynamic response of the floating body. Tuck (1966, 1970) investigated this problem by dividing the computational domain into inner and outer subdomains and the boundary-value problem was solved asymptotically by constructing matched asymptotic expansions in these two subdomains. Yeung (1973) developed a hybrid method which divided the fluid domain into inner and outer regions. The inner region was described by a Rankine source method and in the outer region the radiation potential is expressed in terms of a set of eigenfunctions with unknown coefficients. These unknown coefficients are determined by matching the boundary condition on the interface of the two regions and by satisfying conditions appertaining to finite water depth. Yeung (1973) discovered that there exists a limiting value of the added mass as body motion frequency tends to zero and derived relations illustrating the relationship between damping coefficient and asymptotic wave-amplitude. Bai (1977) applied a similar hybrid method focusing on the behaviour of the hydrodynamic coefficients at zero frequency and concluded that the zero-frequency added mass coefficient is at its minimum value at a critical water depth. Andersen and $\mathrm{He}$ (1985) further improved this hybrid approach to deal with two oscillating bodies with the same or different motion modes, amplitudes and phases. A negative added mass value was observed arising from the interaction effects between the two floating bodies.

In reality, an undulating seabed environment is a more realistic description of a coastal seabed, creating complications to the analysis of water wave problems. The previously described numerical approaches are not directly applicable to the varying bottom contour environments. In the models developed by Buchner (2006); De Hauteclocque et al. (2009); Ferreira and Newman (2009), a second body was introduced to model the sloping seabed. These studies found that the body motion responses are significantly higher in the presence of a sloping seabed than in a constant water depth environment, and it was observed that a clear "wiggle" occurred in the response amplitude operators (RAOs) especially in heave response. In these investigations, the numerical model requires selection of a specific mirrored second body in terms of size and shape which is replicated from case to case, and therefore, it cannot supply a general solution to an arbitrary problem. Athanassoulis and Belibassakis (1999); Belibassakis and Athanassoulis (2004); Belibassakis (2008) adopted a coupled-mode theory to divide the fluid domain into an interior domain of varying water depth and an exterior domain of constant depth. They introduced an extra term called the sloping-bottom mode to correct the incident wave potential so that it satisfies the seabed condition on the portion of the sloping bottom. Numerical predictions of Belibassakis $(2005,2008)$ showed that the sloping seabed does not significantly change the frequency of resonance motion of a freely floating body.

The Rankine source method coupled with other numerical techniques pro- 
vides an approach to deal with various boundary condition problems (Cao et al., 1991; Beck and Scorpio, 1995; Koo and Kim, 2007; Bandyk, 2009; Zhang et al., 2010; Kim and Kim, 2013). In a previous study, Feng et al. (2016) developed a continuous Rankine source model to solve wave-body interaction problems involving various seabed conditions. All the fluid domain surfaces are discretized by continuous panels and the Rankine source integral is calculated analytically on the panel rather than by a numerical approximation as is usually adopted in an isolated source point method. The source panels are placed exactly on the boundary surfaces and therefore numerical errors arising from boundary integral are minimized. Both flat and undulating rigid seabed problems were investigated utilizing a single mathematical model framework. It was found that the mean water depth is a key parameter influencing the hydrodynamic performance of the floating body. It was assumed that the undulating seabed profiles were symmetric and the study focused on the radiation problem involving only heave and sway oscillatory motions.

The far field radiation condition requires careful treatment in the Rankine source method. The infinite free surface domain in such problems requires truncation to a bounded domain but the truncated boundary may cause a reflection wave to propagate backwards to disturb the original wave field. Several numerical techniques are available to avoid wave reflection, amongst which, for example, are approaches involving a numerical beach (Israeli and Orszag, 1981; Kring and Sclavounos, 1995; Kim et al., 1997) and an absorbing layer (Turkel and Yefet, 1998; Filippas and Belibassakis, 2014). These numerical techniques commonly feature the truncation of the infinite free surface, allowing creation of a limited free surface over which source panels are distributed and numerical damping artificially imposed to dampen free surface disturbance until all waves disappear in the far field range. Lee (1992); Zhang and Beck (2007) applied an alternative approach to deal with this reflection problem. They distributed Rankine sources on the free surface in an exponential manner. This permitted modelling of a very large free surface area and, utilizing the periodic characteristic of water waves, numerical computations are completed before the surface wave reaches the truncated boundary. This method was further developed to accommodate wave-seabed-body problems (Feng et al., 2016) and wave-current problems (Feng and Bai, 2016).

In the present study, the continuous Rankine source model is developed further to investigate the seabed environment in both radiation and diffraction problems. This study omits discussion of the adopted numerical techniques including the distribution of source points on the free and body surfaces, the treatment of the intersection points and the free surface time stepping iteration process as these techniques have been fully described elsewhere by Feng et al. $(2015 \mathrm{a}, \mathrm{b})$. Here a new seabed source panel distribution method is developed to deal with an asymmetric seabed profile as well as a flat seabed. The flat seabed environment is investigated for problems not previously covered to better understand the effect of seabed symmetry on water wave problems.

Numerical results show that the water depth $H$ plays an influential role on all hydrodynamic aspects of the water wave problems examined herein except 
the cross coupling coefficients between vertical and horizontal modes of motion. The significance of mean water depth $H$ varies for different hydrodynamic responses. Most hydrodynamic responses increase, however, roll damping coefficient $B_{22}(\omega)$, cross coupling hydrodynamic coefficients $A_{13} / B_{13}$ and $A_{31} / B_{31}$ and moment $M(\omega)$ response in the diffraction problem are decreased as $H$ decreases, whereas, roll added mass coefficient $A_{22}(\omega)$ varies across the motion frequency.

The emphasis of the present study is to investigate the effect of the asymmetric nature of the sloping seabed in both wave radiation and diffraction problems. It is shown that this varies significantly for different hydrodynamic responses. An asymmetry seabed profile has only a modest influence on diagonal hydrodynamic coefficients $A_{11} / B_{11}, A_{22} / B_{22}$ and $A_{33} / B_{33}$ and cross coupling hydrodynamic coefficients $A_{13} / B_{13}$ and $A_{31} / B_{31}$ in the radiation problem. The cross coupling hydrodynamic coefficients $A_{13} / B_{13}, A_{23} / B_{23}, A_{31} / B_{31}$ and $A_{32} / B_{32}$ change significantly from zero values in the case of a flat seabed. Their negative values vary across the frequency range, whereas, their absolute values increase with increasing asymmetry of the seabed topography.

Numerical predictions also demonstrate the symmetric characteristics of hydrodynamic coefficients, $A_{i j}=A_{j i}$ and $B_{i j}=B_{j i}$ in radiation problem regardless of seabed topography. The sloping seabed has a significant influence on force and moment responses in diffraction problem due to wave reflection and shoaling effects, causing an increase of the hydrodynamic responses over a large range of incident wave frequencies. Numerical investigations shows that the horizontal dimension of the sloping seabed and the relative position between sloping seabed and body also have a significant influence on the hydrodynamic responses.

\section{Problem formulation}

\subsection{Formulation of the water wave problem}

Figure 1 illustrates a coordinate frame of reference $O X Z$ with origin $O$ at the centre of a two-dimensional body floating in a fluid domain of finite water depth. The fluid domain $\Omega$ is bounded by a free surface $S_{f}$, body surface $S_{b}$, seabed surface $S_{0}$ and an enclosing surface at infinity, $S_{\infty}$.

The fluid flow is assumed irrotational and its descriptive velocity potential $\phi$ is subject to the Laplace equation

$$
\frac{\partial^{2} \phi}{\partial x^{2}}+\frac{\partial^{2} \phi}{\partial z^{2}}=0 \text { in the fluid domain } \Omega .
$$

For the wave radiation and diffraction problems considered in this paper, the total velocity potential $\phi$ can be expressed in the form:

$$
\phi= \begin{cases}\phi_{R} & \text { for radiation problem } \\ \phi_{I}+\phi_{S} & \text { for diffraction problem }\end{cases}
$$




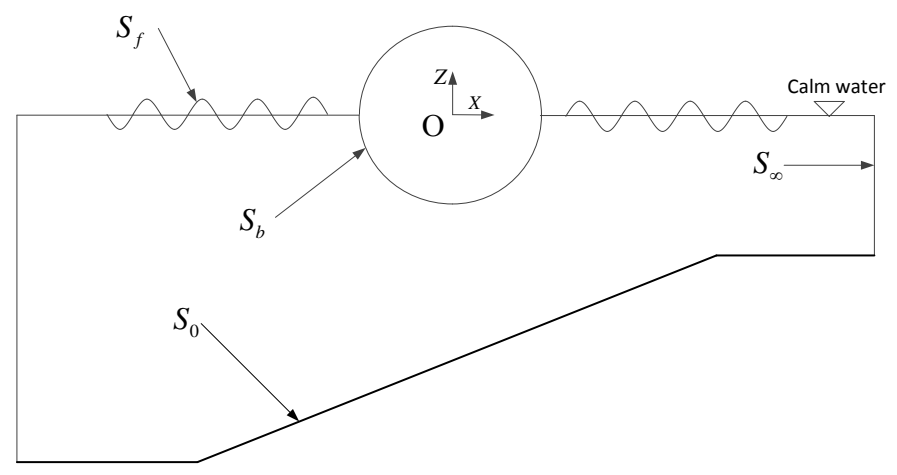

Figure 1: Illustrative sketch of water wave problem.

where $\phi_{I}$ represents the incident wave potential, $\phi_{R}$ the radiation potential and $\phi_{S}$ the scattered wave potential. $\phi_{S}$ can be further expressed as the sum of diffraction potential $\phi_{D}$ and reflection potential $\phi_{r e}$ as:

$$
\phi_{S}=\phi_{D}+\phi_{r e},
$$

where $A$ denotes the incident wave amplitude and $k$ represents the wave number.

For the incoming linear plane progressive wave in a finite water depth $h, \phi_{I}$ is written as

$$
\phi_{I}=\frac{g A}{\omega} \frac{\cosh k(z+h)}{\cosh k h} \sin (k x-\omega t) .
$$

For the wave radiation problem, the associated kinematic body boundary condition is given by

$$
\frac{\partial \phi_{R}}{\partial \mathbf{n}_{b}}=v_{b n} \text { on } S_{b},
$$

where $v_{b n}$ is the body velocity in the normal direction and $\mathbf{n}_{b}$ is the unit normal vector on the body surface pointing out of the fluid domain. In the present study, it is assumed the body experiences forced motion in three degrees of freedom in sway, roll and heave denoted by subscript $i=1,2,3$ respectively. At time $t$, the body velocity $v_{i}(i=1,2,3)$ is described as:

$$
v_{i}=a_{i} \omega \cos \omega t, \quad i=1,2,3
$$


here $a_{i}$ denotes a linear amplitude for $i=1,3$ and an angular amplitude for $i=2$. The frequency $\omega$ represents the oscillatory motion of the body. For the wave diffraction problem, the body boundary condition is expressed as

$$
\frac{\partial \phi_{S}}{\partial \mathbf{n}_{b}}=-\frac{\partial \phi_{I}}{\partial \mathbf{n}_{b}} \text { on } S_{b},
$$

where $v_{b n}$ is the body velocity in normal direction and $\mathbf{n}_{b}$ is the unit normal vector on the body surface pointing out of the fluid domain.

On the solid seabed surface $z=h(x)$, there is no flow penetrates and therefore

$$
\frac{\partial \phi_{R}}{\partial n_{0}}=0 \text { on } S_{0}, z=h(x)
$$

for radiation problem. Here $n_{0}$ is the unit normal vector on the seabed surface pointing out of the fluid domain.

For the diffraction problem, the no flow penetrating boundary condition can be explicitly expressed as:

$$
\frac{\partial \phi_{S}}{\partial n_{0}}=-\frac{\partial \phi_{I}}{\partial n_{0}} \text { on } S_{0}, z=h(x) .
$$

On the parts of the flat seabed, where depth is constant and equal to $h$ (i.e., the depth of the incident wave region which is involved in Eq. 4), it holds that $\frac{\partial \phi_{I}}{\partial n_{0}}=0$ and the seabed boundary condition becomes:

$$
\frac{\partial \phi_{S}}{\partial n_{0}}=0
$$

whereas for other parts of the bottom boundary, including the sloping seabed and other possibly flat horizontal parts of the bottom at different depths, $\frac{\partial \phi_{S}}{\partial n_{0}}$ is non zero and wave reflection occurs.

The linear kinematic and dynamic free surface boundary conditions for both radiation and diffraction problems are given respectively by

$$
\frac{\partial \eta_{k}}{\partial t}-\frac{\partial \phi_{k}}{\partial z}=0 \text { on } S_{f}
$$

and

$$
\frac{\partial \phi_{k}}{\partial t}+g \eta_{k}=0 \text { on } S_{f}
$$

where $\eta$ denotes the elevation of the surface wave and $g$ the gravitational acceleration. The subscript ' $k$ ' stands for ' $R^{\prime}$ or ${ }^{\prime} D$ ' for the radiation or diffraction problem respectively. The boundary condition for the disturbance potential $\phi_{S}$ and $\phi_{R}$ applied on the far field boundary at infinity requires that the generated wave moves towards infinity and never reflects backwards.

The Laplace equation, Eq. 1, together with boundary conditions Eqs. 5, 11,12 and 8 , form a boundary value equation to describe the wave radiation problem whilst the wave diffraction problem is solved by the Laplace equation, Eq. 1 together with boundary conditions, Eqs. 7, 11, 12 and 9. 


\subsection{Numerical discretization}

By utilizing the Gauss divergence theorem, Laplace equation, Eq. 1, and the application of the Dirichlet boundary value problem to the exterior domain of the fluid field, Lamb (1945) [pp.59-60] expresses the velocity potential $\phi$ as a boundary integral of Rankine sources continuously distributed on the fluid boundary $S=S_{b} \cup S_{f} \cup S_{0}$ in the form

$$
\phi\left(\mathbf{x}^{\prime}, t\right)=\int_{S_{b}} \sigma^{b} \ln \left|\mathbf{x}^{\prime}-\mathbf{x}\right| d s_{\mathbf{x}}+\int_{S_{f}} \sigma^{f} \ln \left|\mathbf{x}^{\prime}-\mathbf{x}\right| d s_{\mathbf{x}}+\int_{S_{0}} \sigma^{0} \ln \left|\mathbf{x}^{\prime}-\mathbf{x}\right| d s_{\mathbf{x}} .
$$

Here we denote control point $\mathbf{x}^{\prime}=\left(x^{\prime}, z^{\prime}\right)$, located on the middle point of a continuous panel, the source point $\mathbf{x}=(x, z)$, the body source strength $\sigma^{b}=$ $\sigma\left(\mathbf{x}^{\prime}, t\right)$, free surface source strength $\sigma^{f}=\sigma\left(\mathbf{x}^{\prime}, t\right)$, seabed source strength $\sigma^{0}=$ $\sigma\left(\mathbf{x}^{\prime}, t\right)$ and variable of integration $d s_{\mathbf{x}}$.

It should be noted that, physically, the free surface $S_{f}$ is of infinite length and the integral of this infinite value is not possible by numerical technique. For this reason, the computational domain is truncated in the numerical simulations and the far field boundary condition is satisfied on this truncated free surface. In the present study, the Rankine source panels are distributed in an exponential manner allowing the covering of a very large area of free surface and numerical computations completed before the surface wave reaches the truncated boundary. The interested reader can find detailed information of this Rankine source panel distribution approach in a previous study by Feng et al. (2016).

In the numerical simulation, the fluid domain surface $S$ is discretized by Rankine sources panel rather than isolated Rankine source points. These sources are located exactly on the fluid domain surface $S$ and therefore no desingularized technique is applied. Let the integral surfaces $S_{b}, S_{f}$ and $S_{0}$ be approximated as sums of panels $N_{b}, N_{f}$ and $N_{0}$ respectively such as $S_{b}=\cup_{i=1}^{N_{b}} S_{i}^{b}, S_{f}=\cup_{j=1}^{N_{f}} S_{j}^{f}$ and $S_{0}=\cup_{k=1}^{N_{0}} S_{k}^{0}$.

Therefore, the discretization of Eq. 13 is given by

$$
\begin{aligned}
\phi\left(\mathbf{x}^{\prime}, t\right) & =\sum_{i=1}^{N^{b}} \sigma_{i}^{b} \int_{S_{i}^{b}} \ln \left|\mathbf{x}^{\prime}-\mathbf{x}_{i}\right| d s_{\mathbf{x}} \\
& +\sum_{j=1}^{N^{f}} \sigma_{j}^{f} \int_{S_{j}^{f}} \ln \left|\mathbf{x}^{\prime}-\mathbf{x}_{j}\right| d s_{\mathbf{x}}+\sum_{k=1}^{N^{0}} \sigma_{k}^{0} \int_{S_{k}^{0}} \ln \left|\mathbf{x}^{\prime}-\mathbf{x}_{k}\right| d s_{\mathbf{x}}
\end{aligned}
$$

Figure 2 illustrates the flowchart of the numerical process adopted to solve these boundary value problems. It is noticed that the ramp function, which is usually adopted during the initial time steps to ensure the flow smoothly develops from the initial status, is not used in the present study. Numerical experience demonstrates that numerical instability is not a problem in the developed numerical scheme of study, and the abrupt change in the initial condition, without employing a ramp function, does not lead to any undesirable 
problem. Details of other numerical algorithms were previously presented by Feng et al. (2016) and therefore not repeated here.

The calculation of the influence coefficient matrix accounts for a substantial computational effort. To reduce numerical error and increase computational efficiency, the Rankine source panel integral is calculated analytically in contrast to the algebraic approximation usually adopted in an isolated Rankine source method (Zhang and Beck, 2007; Wang et al., 2015, 2016). For example, on taking the first integral on the right hand side of Eq. 14, the panel integral can be analytically expressed as:

$$
\begin{aligned}
& \sum_{i=1}^{N^{b}} \sigma_{i}^{b} \int_{S_{i}^{b}} \ln \left|\mathbf{x}^{\prime}-\mathbf{x}_{i}\right| d s_{\mathbf{x}}= \\
& \sum_{i=1}^{N^{b}} \sigma_{i}^{b}\left(-L_{b}^{i}-\operatorname{Re}\left(\left(\mathbf{x}^{\prime}-\mathbf{x}_{i+1}\right) \overline{\mathbf{n}}_{b}^{i} \ln \left(\mathbf{x}^{\prime}-\mathbf{x}_{i+1}\right)-\left(\mathbf{x}^{\prime}-\mathbf{x}_{i}\right) \overline{\mathbf{n}}_{b}^{i} \ln \left(\mathbf{x}^{\prime}-\mathbf{x}_{i}\right)\right)\right),
\end{aligned}
$$

where $L_{b}^{i}$ is the length of panel $i$ on body surface and $\overline{\mathbf{n}}_{b}^{i}$ denotes the conjugation of $\mathbf{n}_{b}^{i}$ on this panel. The calculations for the other two integrals on the right hand side of Eq. 14 are represented in a similar manner to Eq. 15.

\subsection{Formulation of hydrodynamic coefficients}

The dynamic pressure on the body surface, $p=p\left(\mathbf{x}_{i}^{\prime b}, t\right)$, is evaluated from Bernoulli's equation expressed as

$$
\frac{p(t)}{\rho}=-\frac{\partial \phi}{\partial t}
$$

where $\rho$ denotes the fluid density. The temporal derivative of the velocity potential $\phi$ is calculated by a finite difference method using the values of the velocity potential at two consecutive time steps.

The dynamic force $F(t)$ and moment $M(t)$ applied to the body surface are calculated by integrating the dynamic pressure over the body surface as given by

$$
\begin{aligned}
& F(t)=\int_{S_{b}} p(t) n_{b} d s, \\
& M(t)=\int_{S_{b}} p(t)\left(\mathbf{r} \times \mathbf{n}_{b}\right) d s,
\end{aligned}
$$

where $\mathbf{r}$ is the position vector of the body surface $d s$.

The application of Fourier series allows the frequency domain added mass $A_{i, j}(\omega),(i, j=1,2,3)$ and damping coefficient $B_{i j}(\omega),(i, j=1,2,3)$ to be expressed in the forms:

$$
A_{i j}(\omega)=\frac{2}{a \omega^{2} T_{0}} \int_{0}^{T_{0}} F_{i j}(t) \sin (\omega t) d t
$$




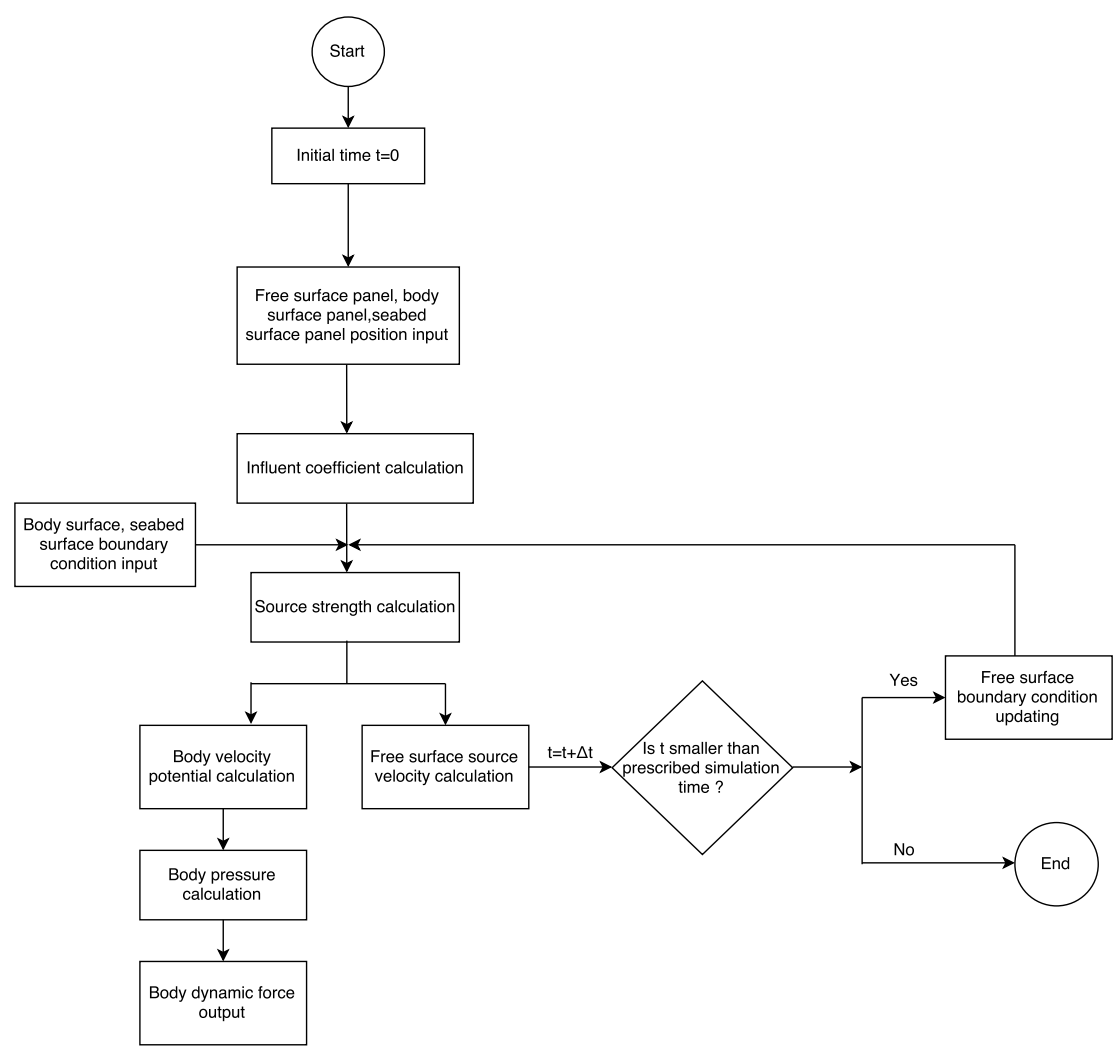

Figure 2: A flowchart of the adopted numerical process.

$$
B_{i j}(\omega)=-\frac{2}{a \omega T_{0}} \int_{0}^{T_{0}} F_{i j}(t) \cos (\omega t) d t,
$$

where $T_{0}$ denotes the period of the body motion. Here $i$ refers to the $i$ th degree of freedom body motion and $j$ to the $j$ th degree of freedom hydrodynamic performance. In this paper $i, j=1,2$ and 3 denote sway, roll and heave motions respectively.

The hydrodynamic coefficient $A_{i j}$ and $B_{i j}$ are represented in the matrix form:

$$
A_{i j}=\left[\begin{array}{lll}
A_{11} & A_{12} & A_{13} \\
A_{21} & A_{22} & A_{23} \\
A_{31} & A_{32} & A_{33}
\end{array}\right] \quad B_{i j}=\left[\begin{array}{lll}
B_{11} & B_{12} & B_{13} \\
B_{21} & B_{22} & B_{23} \\
B_{31} & B_{32} & B_{33}
\end{array}\right] .
$$

The diagonal coefficients $A_{11} / B_{11}, A_{22} / B_{22}$ and $A_{33} / B_{33}$ are the primary coefficients and are grouped as diagonal coefficients for the purpose of simple identification. Off-diagonal coefficients $A_{12} / B_{12}$ and $A_{21} / B_{21}$ are referred as cross 
coupling coefficients. Hydrodynamic coefficients $A_{13} / B_{13}, A_{23} / B_{23}, A_{31} / B_{31}$, and $A_{32} / B_{32}$ are zero for a symmetric body floating in deep water above a flat seabed, but the existence of a sloping seabed destroys this symmetry situation and the evaluation of these coefficients are investigated in this study. These coefficients are classified as vertical and horizontal modes cross coupling hydrodynamic coefficients.

\subsection{Seabed panel distribution}

In the present study the sloping seabed $z=h(x)$ is described mathematically as:

$$
h(x)= \begin{cases}-H-\frac{d}{2} & x<-\frac{L}{2} \\ -H+x \tan \beta & -\frac{L}{2} \leq x \leq \frac{L}{2}, \\ -H+\frac{d}{2} & x>\frac{L}{2}\end{cases}
$$

where $H$ is the mean water depth and $\beta$ denotes the angle of the sloping seabed and defined as:

$$
\beta=\arctan \left(\frac{d}{L}\right) .
$$

As illustrated in Figure 3, $L$ represents the length of the sloping area in the $X$ direction, which is set as $L=6.0 R$ in the following numerical simulations unless stated otherwise, and $R$ denotes the half breadth of the body. $d$ represents the height of the sloping area in the $Z$ direction and is determined by Eq. 23 for a given angle. The sloping seabed is divided into three regions, $h_{1}, h_{2}(x)$ and $h_{3}$. To define the sloping seabed $h_{1}$ and $h_{3}$ correspond to constant water depths on the flat portions either side of the slope and $h_{2}(x)$ denotes the water depth in the region of the slope.

The free surface is divided into inner and outer domains. The Rankine source panels on the left and right sides are arranged in a mirrored manner around the $Z$ axis as illustrated in Figure 3. In the outer domain the distances between the centres of neighbouring source panels are displaced in an exponentially increasing manner. This allows the encompassment of a very large free surface and therefore the generated wave can move towards the far field of this enlarged computational domain without experiencing wave reflections. By this simple and efficient numerical technique, the radiation condition is well satisfied without the need to introduce artificial damping into the mathematical model. The interested reader may consult Feng et al. (2015a) for details of this approach.

Following similar idea as used to describe the free surface, the distribution of seabed source panels is also divided into the inner and outer domains, because the seabed boundary also covers a large area corresponding to the large free surface boundary. However because of the asymmetric nature of the sloping seabed, a mirror image approach is no longer valid and needs adjustment. A parameter $\gamma=1+\tan \beta$ is introduced to deal with this asymmetric characteristic. Starting from $x=0$, on the right hand side of $S_{0}$ the horizontal distances 


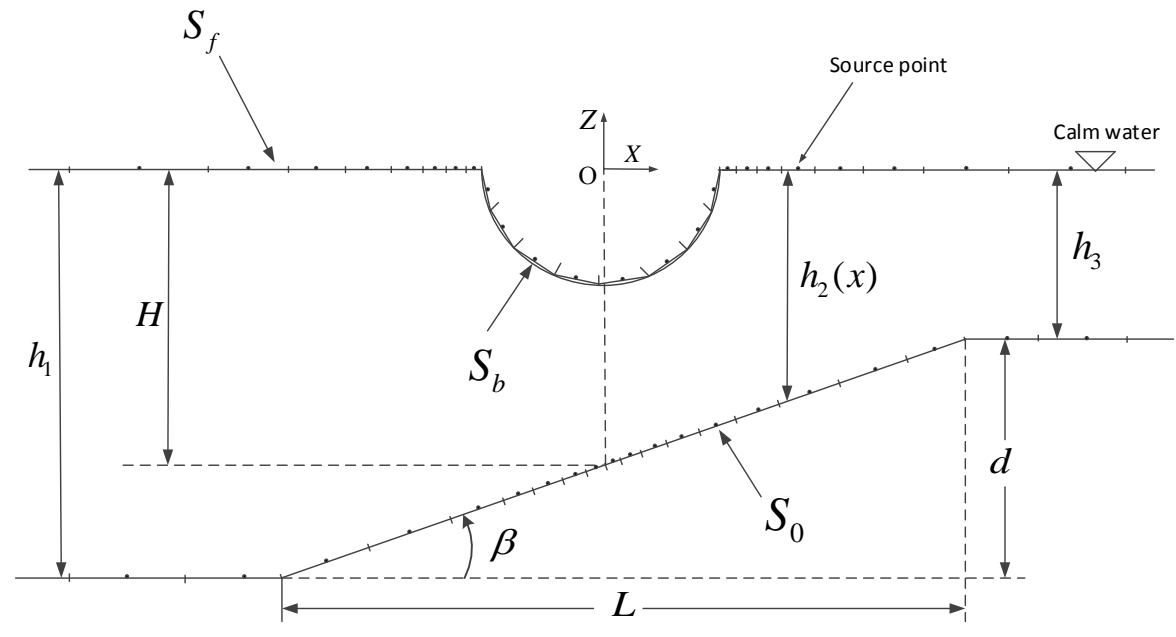

Figure 3: Definition and illustration of the problem under study.

between neighbouring seabed control (source) points $\mathbf{x}_{k}^{\prime 0}$ are expressed in the form:

$$
\begin{aligned}
& \left|\mathbf{x}_{k}^{\prime 0}-\mathbf{x}_{k-1}^{\prime 0}\right|=\frac{L_{b} \alpha_{k}}{\gamma \omega^{2}} \text { in the inner domain, } \\
& \left|\mathbf{x}_{k}^{\prime 0}-\mathbf{x}_{k-1}^{\prime 0}\right|=\frac{L_{b} \alpha_{k}}{\gamma \omega^{2}} 1.05^{k(k-1) / 2} \text { in the outer domain, }
\end{aligned}
$$

and on the left hand side of $S_{0}$ :

$$
\begin{aligned}
& \left|\mathbf{x}^{\prime 0}-\mathbf{x}_{k-1}^{\prime 0}\right|=\frac{\gamma L_{b} \alpha_{k}}{\omega^{2}} \text { in the inner domain, } \\
& \left|\mathbf{x}^{\prime 0}-\mathbf{x}_{k-1}^{\prime 0}\right|=\frac{\gamma L_{b} \alpha_{k}}{\omega^{2}} 1.05^{k(k-1) / 2} \text { in the outer domain, }
\end{aligned}
$$

where $L_{b}$ represents the length of the body surface panel covering the intersection point of the body and free surfaces. The parameter $\alpha_{k}$ defines the separation distances between source points and their values are chosen in the manner described by Feng et al. (2015a). The vertical positions of the control point $\mathbf{x}^{\prime 0}$ are calculated by Eq. 23 once the horizontal positions are established. It is noted that for a flat seabed the angle $\beta=0$ and therefore $\gamma=1$. The sloping seabed panel distribution method applied here is now similar to the one devised for a flat seabed, see Feng et al. (2016). Thus this developed seabed source panel distribution model can deal with both flat and sloping seabed profiles.

\section{Wave reflection evaluation}

Wave reflections occur for various reasons. Section 3.1 discusses wave reflections from the boundary of the numerical domain. These are an undesirable 
phenomenon caused by the truncation of the free surface when positioned too close to the floating body. To avoid these reflections, the proposed method distributes Rankine source panels to cover a very large free surface area so that numerical simulations are completed before the generated surface waves reaches the truncated boundary.

From a physical viewpoint, the presence of a sloping seabed acts, to some degree, as a submersible barrier reflecting/shoaling and modifying the incident wave. No wave reflection occurs when the seabed is flat. Nevertheless, the boundary value equation for this case can be adjusted to include this wave modification by satisfying a sloping seabed boundary condition, as discussed in Section 3.2 .

\subsection{Wave reflection from truncated computational domain}

In order to demonstrate the efficiency of the proposed free surface source distribution approach for sloping seabed cases, numerical tests are performed for a circular cylinder experiencing forced heave motion above a sloping seabed environment with mean water depth $H=2.0 R$ and slop angle $\beta=\arctan 1 / 4$. Three types of free surface source point distribution methods are examined. These are referred to as Type 1 and 2 with source points covering 1 and 2 wave lengths on one side of the free surface and Type 3 using the proposed method as discussed in Section 2.4.

Figure 4 shows the time record of heave force $F_{z}(t)$ for these three different types of free surface source distributions. It is observed that the wave reflections occur and disturb the force profile significantly for Types 1 and 2 and earlier reflections appear in the smaller free surface range. In contrast, in the proposed method, nearly 100 wave lengths are covered on one side of the free surface and therefore the generated wave moves towards infinity without noticeable reflections appearing on the free surface during the numerical simulation.

The proposed numerical model is also applicable to an arbitrary bottom topography extending to infinity. To demonstrate this, a new sloping seabed is introduced in which the right hand side of the flat seabed portion $h_{3}=-H+\frac{d}{2}$ is replaced by a rippled seabed extending to the far field as defined by

$$
h_{3}(x)=-H+\frac{d}{2}+\frac{1}{10} d \sin \left(x-\frac{L}{2}\right) .
$$

Other information remains unchanged compared to the case introduced at the beginning of the section.

Figure 5 illustrates sketches of these seabed configurations extending to infinity, namely $(a)$ a sloping seabed with flat seabed and constant water depth, and $(b)$ a sloping seabed with rippled seabed profile and varying water depth. For these environments, Figure 6 shows the predicted heave force $F_{z}(t)$ determined by the proposed numerical model. It is observed that the rippled seabed has only minor influence on the predicted results. This numerical test further validates the effectiveness of the free surface source panel distribution for any sloping seabed extending to infinity. It should be emphasised that the rippled 


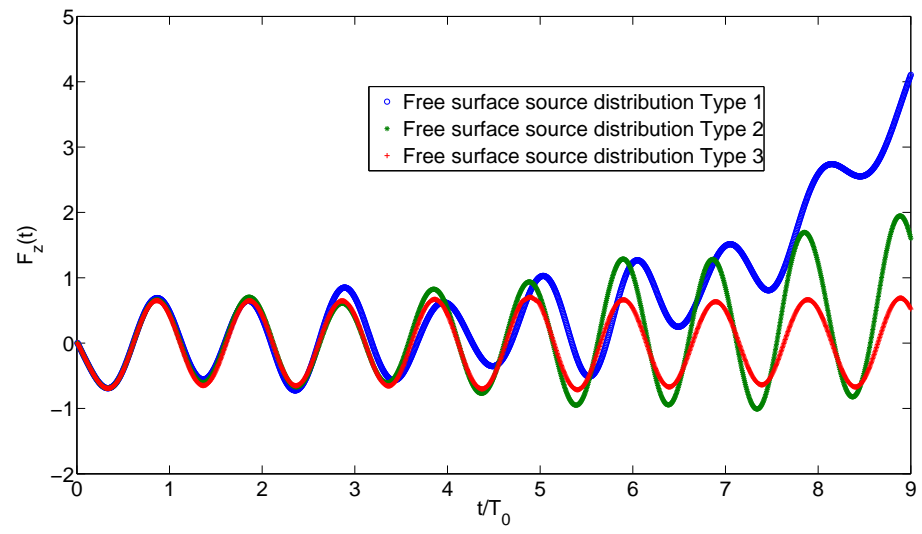

Figure 4: Comparisons of the calculated heave force $F_{z}(t)$ associated with an oscillating circular cylinder adopting three different free surface source distributions (Types $1,2,3$ ). The sloping seabed is of mean water depth $H=2.0 R$ and slope angle $\beta=\arctan 1 / 4$.

seabed has limited effect on the wave reflection because it is located in a relatively deep position and has a small curvature shape, and also the incident wave frequency is far from the Bragg resonance of rippled seabed. The wave reflection becomes more obvious if the mean water depth is reduced and/ or the amplitude of seabed bottom corrugations is increased and/or the incident wave frequency becomes closer to the Bragg resonance of rippled seabed.

It is noted that the seabed source panel distribution described in Eqs. 24 and 25 are not adopted for the rippled seabed, as this approach provides insufficient resolution to define the panels representing the seabed geometry. In such situation, many more seabed source panels are used to define details of the irregular, infinite, rippled seabed. 


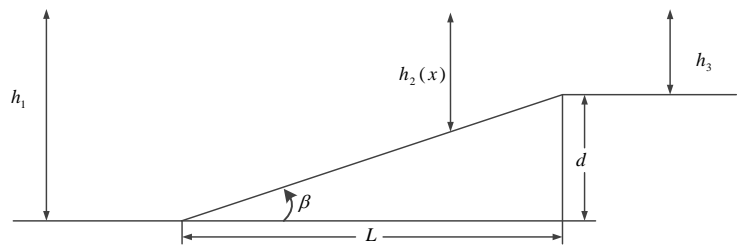

(a)

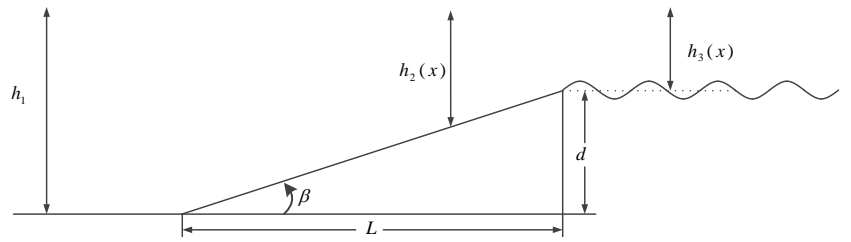

(b)

Figure 5: Sketch of seabed configurations extending to infinity, $(a)$ a sloping seabed with flat seabed at constant water depth and $(b)$ a sloping seabed with varying seabed profile and water depth.

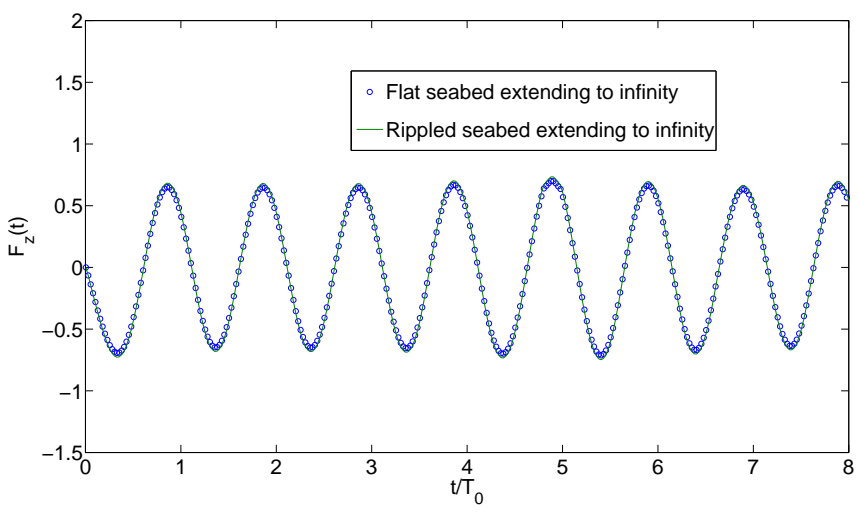

Figure 6: Comparisons of heave force $F_{z}(t)$ associated with a circular cylinder oscillating above a flat seabed and a rippled seabed extending to infinity. 


\subsection{Wave reflection off a sloping seabed}

The presence of a sloping seabed cause wave reflection for both radiation and diffraction problems. These wave reflection effects are accounted for in the mathematical model by satisfying Eqs. 8 and 9 for wave radiation and diffraction problems. To treat this wave reflection phenomenon through an alternative analysis, a reflection potential $\phi_{r e}$ is introduced to amend seabed boundary condition. The total velocity potential $\phi$ for diffraction problem is expressed as:

$$
\phi=\phi_{I}+\phi_{D}+\phi_{r e} .
$$

Here the diffracted wave potential $\phi_{D}$ can be solved through the boundary integral equations as described for $\phi_{S}$ in Section 2.1 with the exception that the sloping seabed condition, Eq. 9, becomes

$$
\frac{\partial \phi_{D}}{\partial n_{0}}=0 \text { on } S_{0}, z=h(x) .
$$

as wave reflection is accounted for by the reflection potential $\phi_{r e}$. The wave refection effect caused by the sloping seabed is accounted here solely by the wave reflection potential $\phi_{r e}$ while this effect is considered by $\phi_{S}$ using sloping seabed boundary condition, Eq. 9.

$\phi_{r e}$ satisfies the Laplace equation, Eq. 1 and is determined by solving boundary value equations constructed by:

$$
\begin{aligned}
& \frac{\partial \phi_{r e}}{\partial n_{b}}=0 \text { on } S_{b}, \\
& \frac{\partial \eta}{\partial t}-\frac{\partial \phi_{r e}}{\partial z}=0 \text { on } S_{f}, \\
& \frac{\partial \phi_{r e}}{\partial t}+g \eta=0 \text { on } S_{f}, \\
& \frac{\partial \phi_{r e}}{\partial n_{0}}=-\frac{\partial \phi_{I}}{\partial n_{0}} \text { on } S_{0}, z=h(x) .
\end{aligned}
$$

It might be argued that the incident wave amplitude $A$ and wave number $k$ are varying on the sloping seabed. In fact, the wave reflection from the sloping seabed (or the influence of the changing seabed geometry on the linear wave at the constant water depth) is accounted by the bottom boundary condition (Eq. 9 or Eq. 34). This treatment is equivalent to the consideration of wave diffraction around two bodies. In this case, the scattered potential $\phi_{S}$ is the total diffraction potential, caused by the fixed body (the first body) and the sloping seabed (the second body) located at the constant water depth, and Eq. 9 or Eq. 34 become the body boundary condition for the second body in this diffraction problem

The combined reflection potential $\phi_{r e}$ and diffraction potential $\phi_{D}$ produce exactly the same results as the scattered potential $\phi_{S}$ proposed in Section 2.1. 
This demonstrates that the incident wave reflection effect can be accounted for by scattered potential $\phi_{S}$ satisfying seabed boundary condition, Eq. 9.

The reflected wave moves towards the left hand side of the fluid domain and possibly transforms into a free surface wave travelling into the far field. As the proposed free surface source distribution method covers a very large area of free surface on both left and right hand sides of the body, this generated free surface wave will not be reflected backwards to disturb the fluid domain.

\section{Numerical results}

To illustrate the developed mathematical model, a circular cylinder of beam $(B=2 R)$ to draft $(T)$, ratio $B / T=2$ and a rectangular cylinder of beam $(B=$ $2 R)$ to draft $(T)$, ratio $B / T=2$ are adopted for numerical simulations. The free surface boundary condition is satisfied on the calm water surface and the body boundary condition is satisfied on the mean wetted body surface because the chosen amplitude of the oscillatory motion is assumed small.

\subsection{Wave radiation problem involving flat or sloping seabed profiles}

To demonstrate the numerical convergence of the proposed method, numerical simulations were performed for the circular cylinder experiencing forced heave motion over a sloping seabed of slope angle $\beta=\arctan 1 / 4$. The numerical results show that this seabed profile discretized by 40 panels on the right and left hand sides of the cylinder produces the equivalent level of numerical accuracy as using 120 panels in the previous method developed for a flat seabed, see Feng et al. (2016). This indicates the proposed method is only slightly more computationally intensive in comparison to the deep water boundary value problems examined previously.

\subsubsection{Seabed effect on diagonal hydrodynamic coefficients}

Added mass coefficient $A_{i j}$ and damping coefficient $B_{i j}$ are the two principle coefficients defining the hydrodynamic characteristics of wave radiation problems. Figures 7 and 8 demonstrate comparisons of heave added mass coefficient $A_{33}(a)$, damping coefficient $B_{33}(b)$ and sway added mass coefficient $A_{11}(a)$, damping coefficient $B_{11}(b)$ for a flat seabed at depth $H=2 T$ and a sloping seabed of angle $\beta=\arctan 1 / 6,1 / 5$ and $1 / 4$ respectively. To demonstrate the effect of the sloping seabed which creates an overall asymmetric profile, the mean water depth under the cylinder is the same in all cases. This condition is assumed in the following simulation unless otherwise stated. From the presented evidence it is seen that the sloping seabed has little effect on the coefficients $A_{11}$, $B_{11}, A_{33}$ and $B_{33}$.

Numerical simulations associated with the rectangular cylinder experiencing forced rolling motion are shown in Figure 9. This shows a comparison of the roll added inertia coefficient $A_{22}(a)$ and the roll damping coefficient $B_{22}(b)$ in deep water $H=\infty$, a flat seabed under water depths of $H=2.0 T$ and $H=1.5 T$ together with the numerical predictions of Zhang and Beck (2007). 
The good agreement between all these calculated results provides a limited measure of validation of the present numerical model for roll motion. It can be seen that seabed imposes large hydrodynamic effects on the added inertia coefficient $A_{22}$, causing it to decrease in value as water depth $H$ decrease for frequency $\omega^{2} B / 2 g \leq 0.7$ and increasing with a decrease in $H$ when motion frequency $\omega^{2} B / 2 g>0.7$. This is in line with the finding of Kim (1969) who adopted a multipole expansion method in the frequency domain. The damping coefficient $B_{22}$ decreases in value as the water depth becomes shallower.

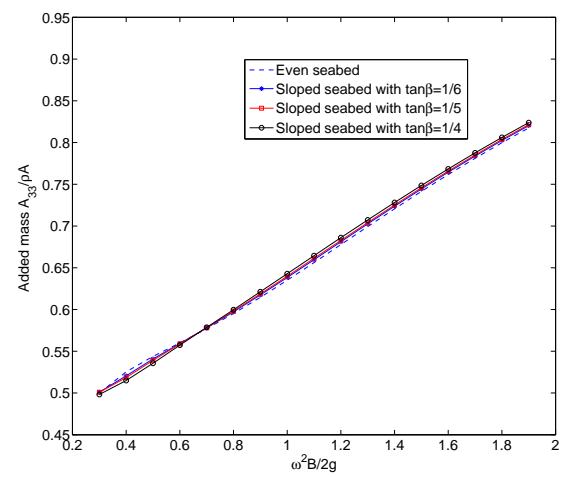

(a) Heave added mass coefficient $A_{33}(\omega)$

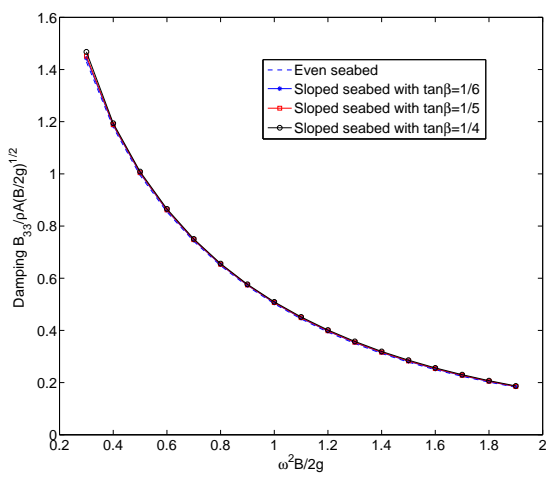

(b) Heave damping coefficient $B_{33}(\omega)$

Figure 7: Comparison of heave added mass coefficient $A_{33}$ (a) and heave damping coefficient $B_{33}$ (b) for a flat seabed in water depth $H=2 T$ and a sloping seabed of slope angle $\beta=$ $\arctan 1 / 6,1 / 5$ and $1 / 4$ respectively.

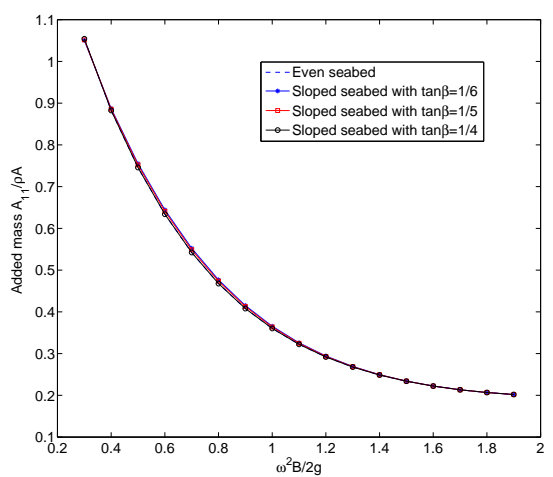

(a) Sway added mass coefficient $A_{11}(\omega)$

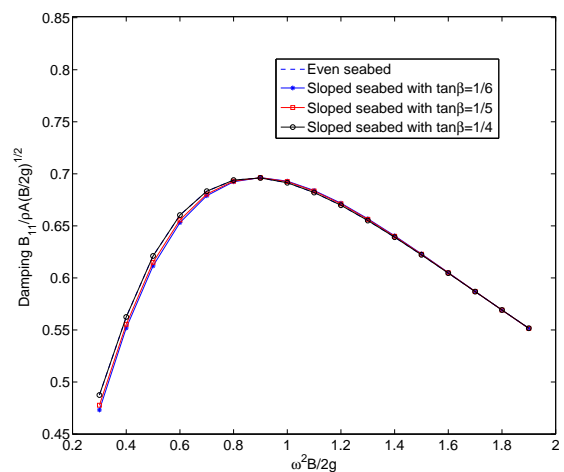

(b) Sway damping coefficient $B_{11}(\omega)$

Figure 8: Comparison of sway added mass coefficient $A_{11}(\omega)$ (a) and sway damping coefficient $B_{11}(\omega)$ (b) for a flat seabed at depth $H=2 T$ and a sloping seabed of slope angle $\beta=$ $\arctan 1 / 6,1 / 5$ and $1 / 4$ respectively. 


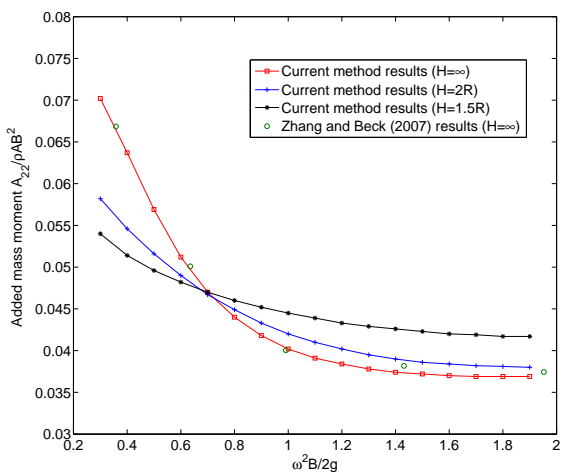

(a) Roll added inertia coefficient $A_{22}(\omega)$

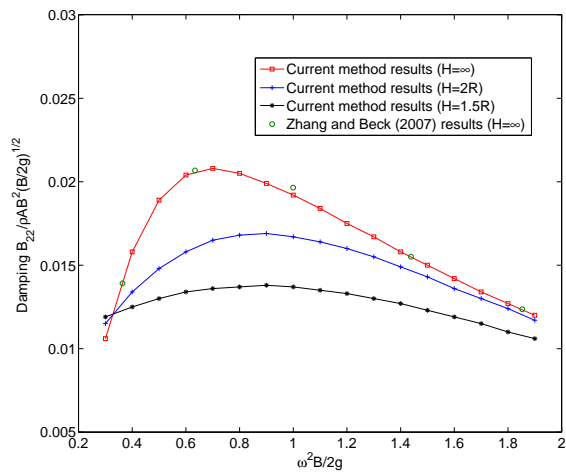

(b) Roll damping coefficient $B_{22}(\omega)$

Figure 9: Comparison of roll added inertial coefficient $A_{22}$ (a) and roll damping coefficient $B_{22}$ (b) between results from the proposed method for a flat seabed at depths $H=\infty, H=2.0 R$ and $H=1.5 R$ and those of Zhang and Beck (2007) for seabed depth $H=\infty$.

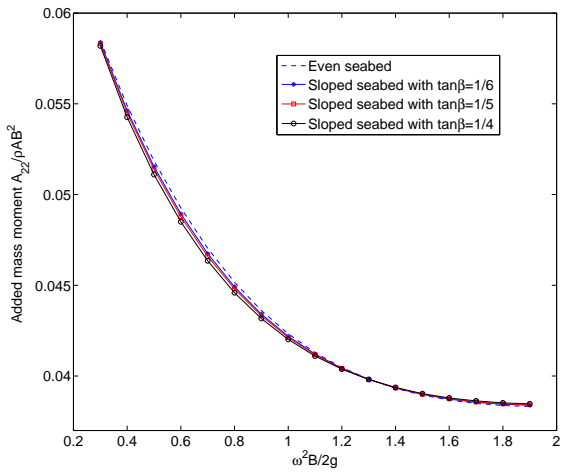

(a) Roll added inertial coefficient $A_{22}(\omega)$

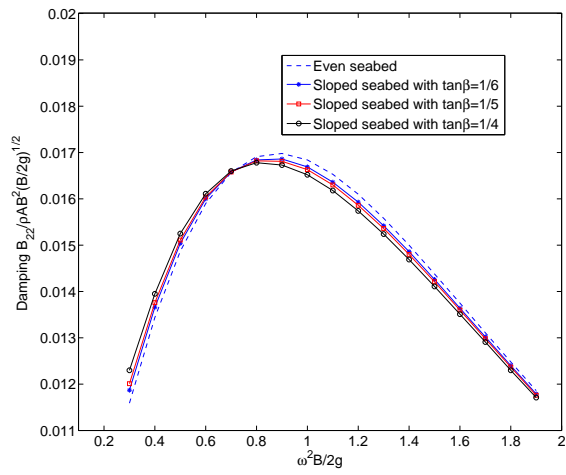

(b) Roll damping coefficient $B_{22}(\omega)$

Figure 10: Comparison of roll added inertia coefficient $A_{22}$ (a) and roll damping coefficient $B_{22}$ (b) for a flat seabed at depth $H=2 T$ and a sloping seabed of slope angle $\beta=\arctan 1 / 6$, $1 / 5$ and $1 / 4$ respectively.

Figure 10 shows a comparison of roll added inertia coefficient $A_{22}(a)$ and the damping coefficient $B_{22}(b)$ for a flat seabed at depth $H=2 T$ and a sloping seabed of slope angle $\beta=\arctan 1 / 6,1 / 5$ and $1 / 4$ respectively. This comparison shows that the asymmetric seabed profile has minor effect on the hydrodynamic characteristics associated with roll motion. It is concluded that a sloping seabed has little influence on the value of the diagonal hydrodynamic coefficients. In a previous study, Feng et al. (2016) demonstrated the importance of mean water depth in determining the hydrodynamic characteristics of a body experiencing linear motion in sway or heave over the profile of a symmetric seabed. This 
conclusion now extends to a wider range of seabed profiles for both linear and angular (roll) motions.

\subsubsection{Seabed effect on cross coupling hydrodynamic coefficients}

Further numerical simulations were performed to investigate the seabed effect on the cross coupling hydrodynamic coefficients. Figure 11 shows data for the roll-sway added mass coefficient $A_{21}(a)$ and damping coefficient $B_{21}$ (b). These predictions by the proposed method are for seabed depths $H=\infty$, $H=2.0 T$ and $H=1.5 T$ and are compared to the numerical and experimental data of Vugts (1968) for water depth $H=\infty$. This comparison illustrates reasonable agreement and provides a limited degree of validation of the proposed method for the calculation of these cross coupling hydrodynamic coefficients.

The comparison also demonstrates that the seabed has a significant effect on both added mass coefficient $A_{21}$ and damping coefficient $B_{21}$. Both these coefficients show negative values. For motion frequency $\omega^{2} B / 2 g \leq 1.0$, the added mass coefficient $A_{21}$ is characterized by a decreasing value as mean water depth decreases but, above this frequency value, it tends to a constant regardless of the value of water depth $H$. The damping coefficient $B_{21}$ also shows a decreasing value with decreasing mean water depth $H$ except in the low motion frequency region, i.e. $\omega^{2} B / 2 g<0.4$.

Figure 12 illustrates a comparison of added mass coefficient $A_{21}$ (a) and damping coefficient $B_{21}$ (b) for a flat seabed at $H=2 T$ and a sloping seabed with slope angle $\beta=\arctan 1 / 6,1 / 5$ and $1 / 4$ respectively. It is observed that the sloping seabed has little effect on these hydrodynamic coefficients.

Figure 13 shows comparisons of sway-roll added mass coefficient $A_{12}(a)$ and damping coefficient $B_{12}(b)$ for a flat seabed at depths $H=\infty, H=2.0 T$ and $H=1.5 T$ whereas Figure 14 illustrates comparisons of these coefficients for a flat seabed at $H=2 T$ and a sloping seabed of slope angle $\beta=\arctan 1 / 6$, $1 / 5$ and $1 / 4$ respectively. Similar conclusions are deduced for the behaviour of these coefficients associated with sway-roll as for roll-sway demonstrating that $A_{12}=A_{21}$ and $B_{12}=B_{21}$ provided that the same seabed condition applies. Vugts (1970); Newman (1977) [pp.140-141] proved mathematically that the hydrodynamic coefficients $A_{i j}=A_{j i}$ and $B_{i j}=B_{j i}$ for symmetric body. The presented numerical simulations further confirm these conclusions for a flat or sloping seabed environment.

\subsubsection{Seabed effect on vertical and horizontal modes cross coupling hydrody- namic coefficients}

For a symmetric body in deep water or in finite water depth with a symmetric seabed topography the vertical and horizontal modes cross coupling hydrodynamic coefficients are zero. However, a sloping seabed creates an asymmetric topology invalidating this result. Figure 15 shows a comparison of heavesway added mass coefficient $A_{31}(a)$ and heave-sway damping coefficient $B_{31}$ (b) for a flat seabed at depth $H=2 T$ and a sloping seabed with slope angle $\beta=\arctan 1 / 6,1 / 5$ and $1 / 4$ respectively. The value of $A_{31}$ and $B_{31}$ are sig- 


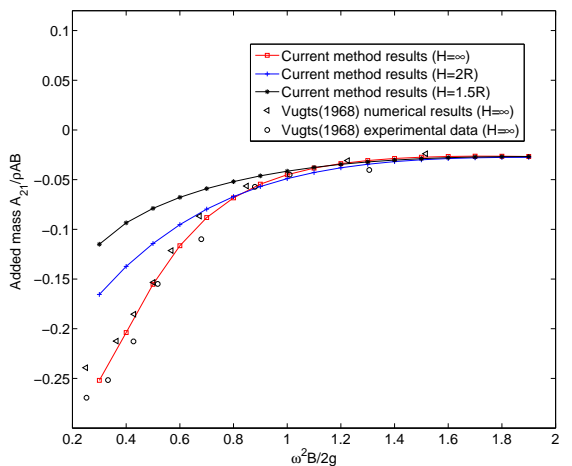

(a) Roll-sway added mass coefficient $A_{21}(\omega)$

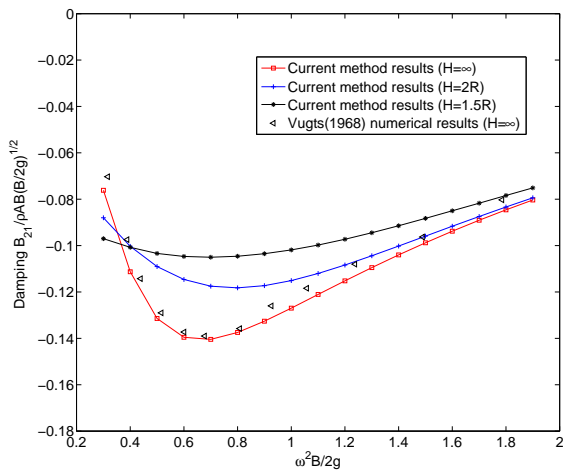

(b) Roll-sway damping coefficient $B_{21}(\omega)$

Figure 11: Comparison of roll-sway added mass coefficient $A_{21}$ (a) and roll-sway damping coefficient $B_{21}$ (b) determined by the proposed method for a flat seabed at depths $H=\infty$, $H=2.0 T$ and $H=1.5 T$ and the numerical and experimental data of Vugts (1968) for seabed depth $H=\infty$.

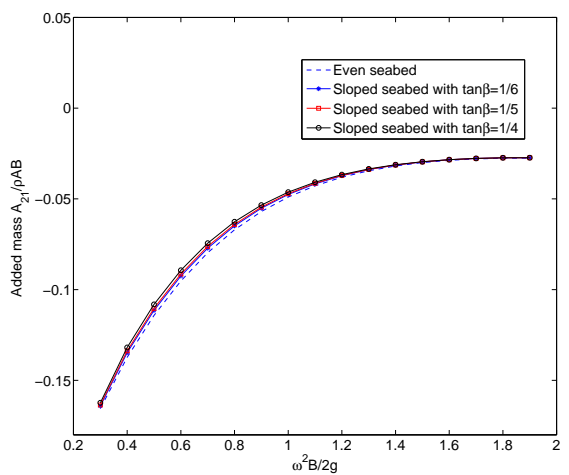

(a) Roll-sway added mass coefficient $A_{21}(\omega)$

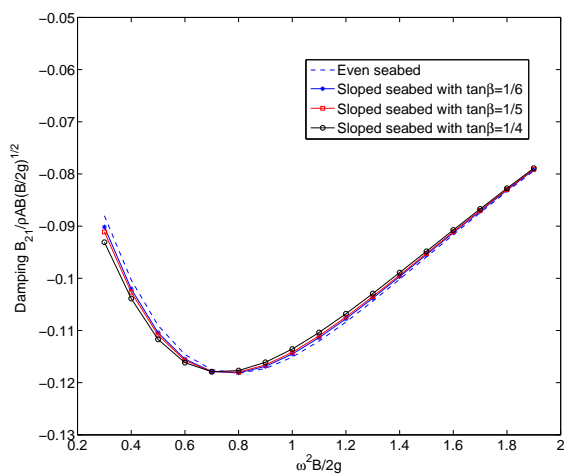

(b) Roll-sway damping coefficient $B_{21}(\omega)$

Figure 12: Comparison of roll-sway added mass coefficient $A_{21}$ (a) and roll-sway damping coefficient $B_{21}$ (b) predicted by the proposed method for a flat seabed at depth $H=2 T$ and a sloping seabed of slope angle $\beta=\arctan 1 / 6,1 / 5$ and $1 / 4$ respectively.

nificant when motion frequency $\omega^{2} B / 2 g \leq 1.0$ and both these values increases with increasing slope angle $\beta$.

Figure 16 shows a comparison of heave-sway added mass coefficient $A_{13}(a)$ and heave-sway damping coefficient $B_{13}(b)$ for a flat seabed at water depth $H=2 T$ and a sloping seabed with slope angle $\beta=\arctan 1 / 6,1 / 5$ and $1 / 4$ respectively. The same conclusion is deduced for the characteristic behaviour of sway-heave as for heave-sway and it is observed that $A_{31}=A_{13}$ and $B_{31}=B_{13}$ provided the same seabed condition applies. 


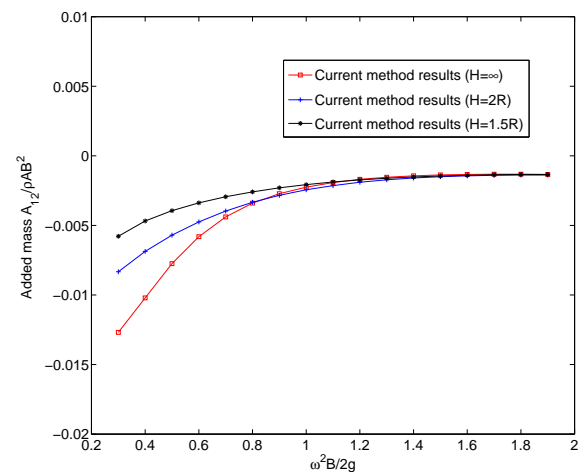

(a) Sway-roll added mass coefficient $A_{12}(\omega)$

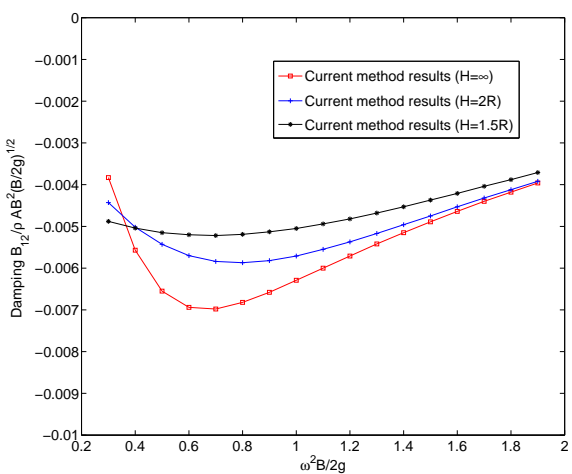

(b) Sway-roll damping coefficient $B_{12}(\omega)$

Figure 13: Comparison of sway-roll added mass coefficient $A_{12}$ (a) and sway-roll damping coefficient $B_{12}$ (b) predicted by the proposed method for a flat seabed at depths $H=\infty$, $H=2.0 T$ and $H=1.5 T$ respectively.

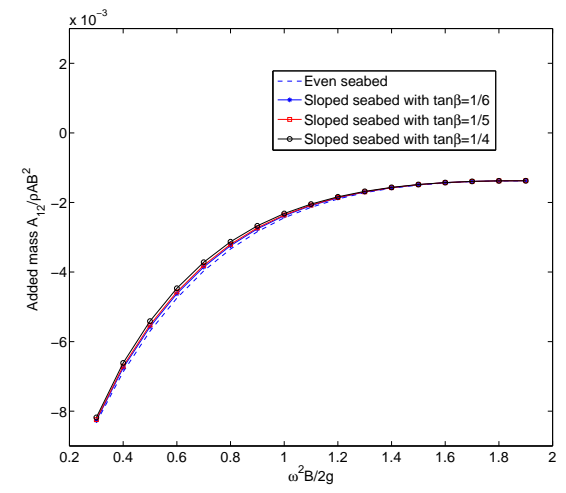

(a) Sway-roll added mass coefficient $A_{12}(\omega)$

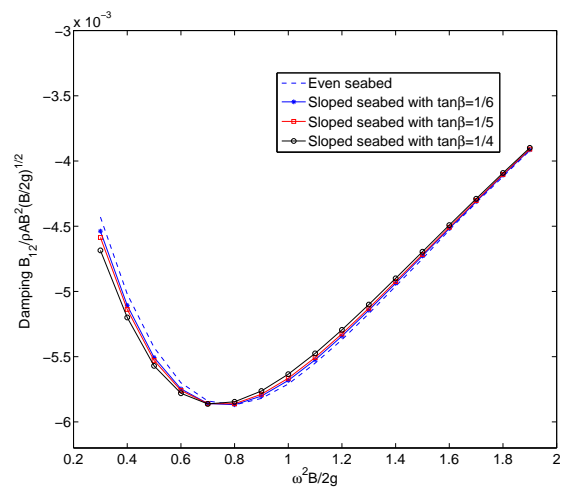

(b) Sway-roll damping coefficient $B_{12}(\omega)$

Figure 14: Comparison of sway-roll added mass coefficient $A_{12}$ (a) and sway-roll damping coefficient $B_{12}$ (b) predicted by the proposed method for a flat seabed at depth $H=2 T$ and a sloping seabed of slope angle $\beta=\arctan 1 / 6,1 / 5$ and $1 / 4$ respectively.

Adopting the same seabed conditions as described previously, Figure 17 illustrates a comparison of roll-heave added mass coefficient $A_{23}(a)$ and damping coefficient $B_{23}(b)$. Both $A_{23}$ and $B_{23}$ are of significant values with $A_{23}$ remaining negative across the frequency range whereas $B_{23}$ crosses the positive-negative barrier at an approximate motion frequency $\omega^{2} B / 2 g=0.9$. A sloping seabed causes an increasing effect on the roll-heave coefficients with increasing slope angle $\beta$. Again the same findings are deduced for heave-roll as for roll-heave as shown in Figure 18 with $A_{32}=A_{23}$ and $B_{32}=B_{23}$ for the same seabed topography. 

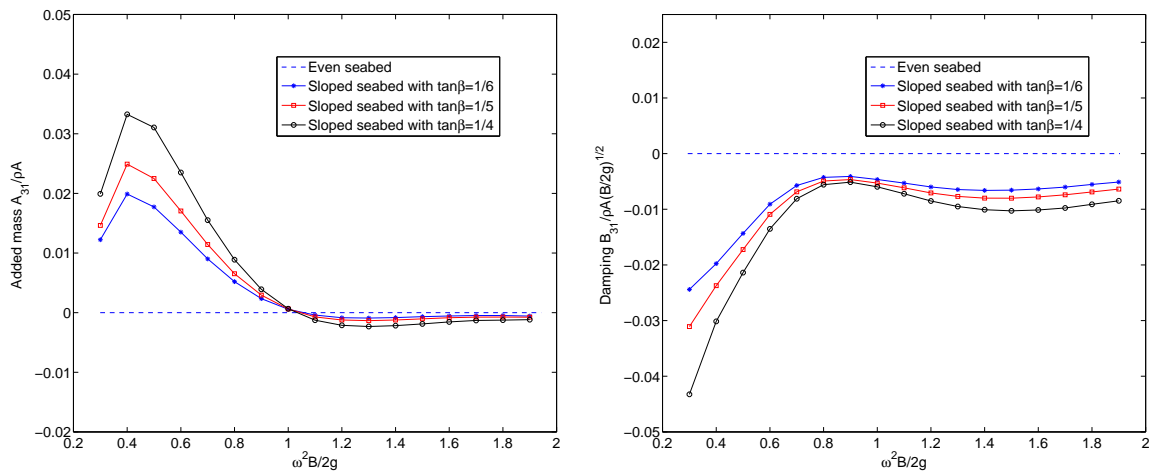

(a) Heave-sway added mass coefficient $A_{31}(\omega)$ (b) Heave-sway damping coefficient $B_{31}(\omega)$

Figure 15: Comparison of heave-sway added mass coefficient $A_{32}$ (a) and heave-sway damping coefficient $B_{32}$ (b) for a flat seabed at depth $H=2 T$ and a sloping seabed of slope angle $\beta=\arctan 1 / 6,1 / 5$ and $1 / 4$ respectively.
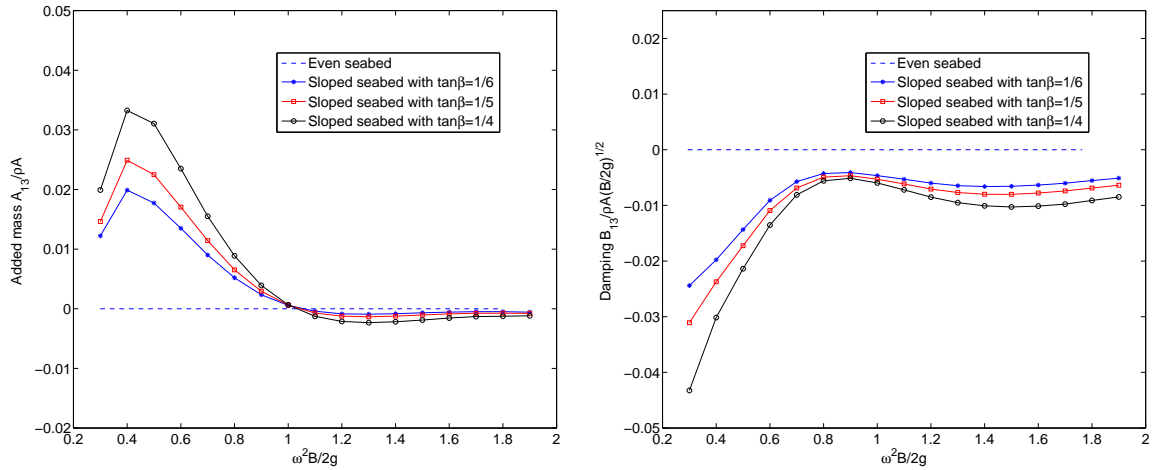

(a) Sway-heave added mass coefficient $A_{13}(\omega)$ (b) Sway-heave damping coefficient $B_{13}(\omega)$

Figure 16: Comparison of sway-heave added mass coefficient $A_{13}$ (a) and sway-heave damping coefficient $B_{13}$ (b) for a flat seabed at depth $H=2 T$ and a sloping seabed of slope angle $\beta=\arctan 1 / 6,1 / 5$ and $1 / 4$ respectively. 


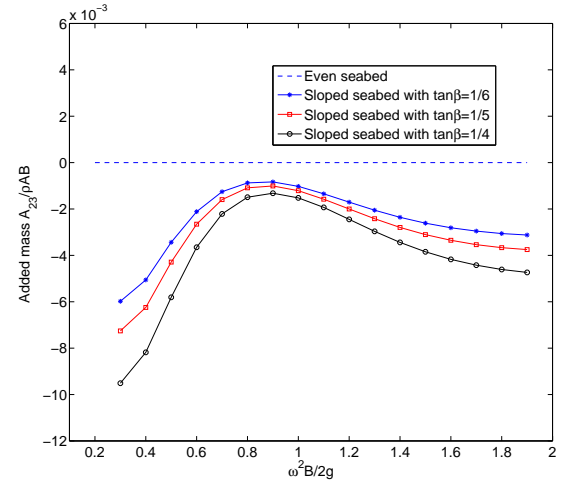

(a) Roll-heave added mass coefficient $A_{23}(\omega)$

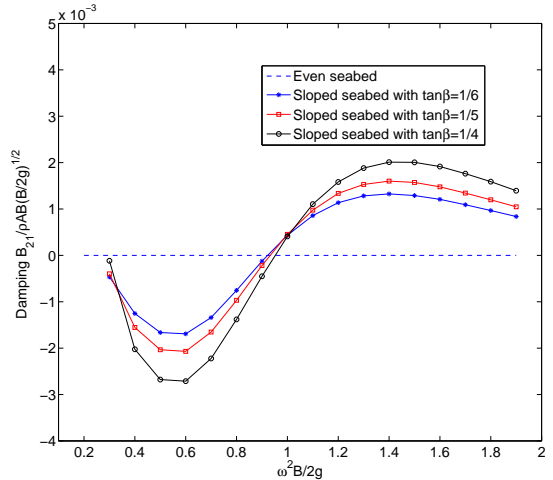

(b) Roll-heave damping coefficient $B_{23}(\omega)$

Figure 17: Comparison of roll-heave added mass coefficient $A_{23}$ (a) and roll-heave damping coefficient $B_{23}$ (b) for a flat seabed at depth $H=2 T$ and a sloping seabed of slope angle $\beta=\arctan 1 / 6,1 / 5$ and $1 / 4$ respectively.

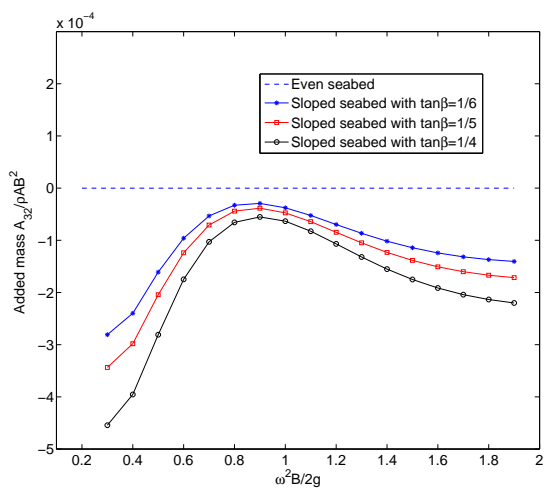

(a) Heave-roll added mass coefficient $A_{32}(\omega)$

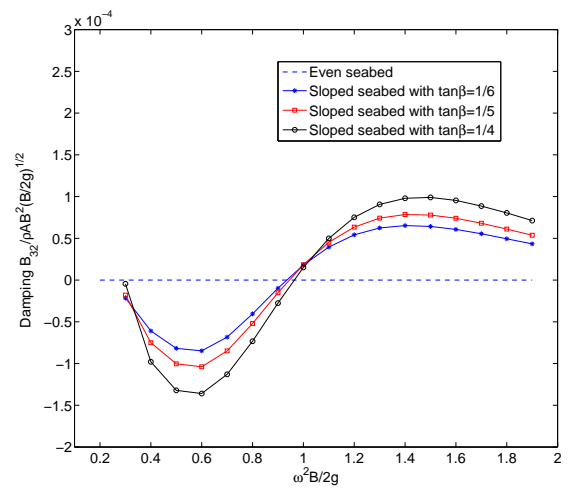

(b) Heave-roll damping coefficient $B_{32}(\omega)$

Figure 18: Comparison of heave-roll added mass coefficient $A_{32}$ (a) and heave-roll damping coefficient $B_{32}$ (b) for a flat seabed at depth $H=2 T$ and a sloping seabed of slope angle $\beta=\arctan 1 / 6,1 / 5$ and $1 / 4$ respectively. 


\subsection{Wave diffraction problem involving flat and sloped seabeds}

In this section the wave diffraction problem is investigated utilizing the circular and rectangular cylinders. The total diffraction force $F$ or moment $M$ is the sum of Froude-Krylov force and the diffraction exciting force. The discretization of the whole fluid domain remains the same as used in the radiation problem.

The existence of sloping seabed introduces an extra reflecting wave. Its contribution is accounted for in the mathematical model by the proposed boundary value equations with the sloping seabed condition applied to the diffraction wave potential $\phi_{D}$ or, by introducing an extra reflection potential and constructing a corresponding boundary value equations. As discussed in section 3.2, both these two methods generate same results. Only one boundary value equation system is required to solve in the former model whilst the latter method requires solution of two equation systems. The proposed numerical method benefits by reduced computational cost and the following results are derived using the one equation system.

For the circular cylinder, Figure 20 illustrates a comparison of the calculated horizontal force $F_{x}(\omega)$ (a) and vertical force $F_{z}(\omega)$ (b) using the proposed method for water depths $H=\infty, H=2.0 R$ and $H=1.5 R$ and compared to the numerical predictions of Zhao and Faltinsen (1988) for water depth $H=\infty$. This comparison provides only a limited validation of the mathematical solution to the diffraction problem although it demonstrates the influence of the seabed on the diffraction force which increases as water depth $H$ decreases over the incident wave frequency range.

Figure 21 shows comparisons of the horizontal force $F_{x}(\omega)$ (a) and the vertical force $F_{z}(\omega)$ (b) for a flat seabed at depth $H=2 T$ and a sloping seabed of slope angle $\beta=\arctan 1 / 6,1 / 5$ and $1 / 4$ respectively. The sloping seabed increases the force responses particularly in the frequency range $0.3<\omega^{2} B / 2 g<1.2$. However, this increase level gradually reduces as the wave frequency $\omega^{2} B / 2 g$ increases and the effect is very small when wave frequency $\omega^{2} B / 2 g>1.5$. These conclusions support the findings of Kim and Kim (2013).

For the rectangular cylinder in water depths $H=\infty, H=2.0 R$ and $H=$ $1.5 R$ respectively, comparisons of numerical simulations of moment $M(\omega)$ are shown in Figure 22. It is observed that the presence of the seabed significantly affects the roll response with the roll moment $M(\omega)$ magnitude reducing with decreasing water depth $H$ for incident wave frequency $\omega^{2} B / 2 g>0.4$. Figure 23 shows a comparison of the moment $M(\omega)$ for a flat seabed at depth $H=2 T$ and a sloping seabed of slope angle $\beta=\arctan 1 / 6,1 / 5$ and $1 / 4$ respectively. This evidence shows that the asymmetry of the sloping seabed has significant influence on the roll moment when wave frequency $\omega^{2} B / 2 g<1.5$.

\subsection{Influence of relative position between body and sloping seabed}

In all the earlier sloping seabed investigations, the distance of the base of the sloping seabed in the horizontal direction, $L$, is uniformly set as $L=6.0 \mathrm{R}$ (see Fig. 3) and the body centred at the middle of the sloped seabed. In order 


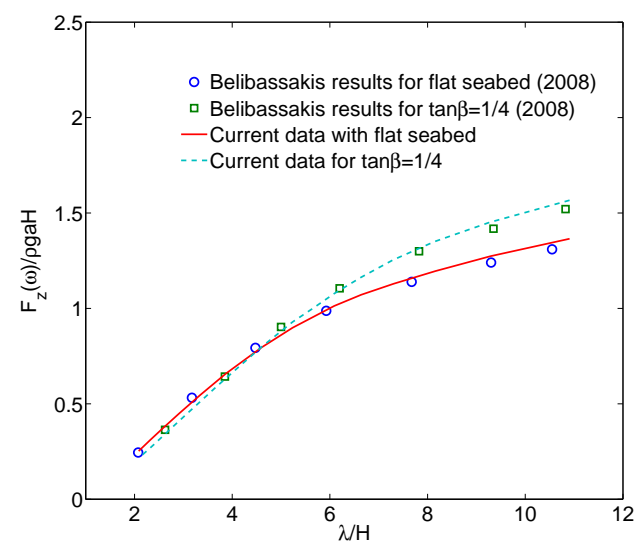

Figure 19: Comparison of moment $M(\omega)$ for water depths $H=\infty, H=2.0 R$ and $H=1.5 R$ respectively.

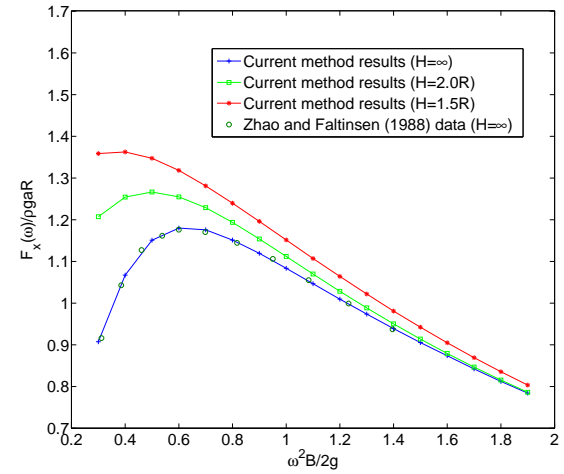

(a) Horizontal force $F_{x}(\omega)$

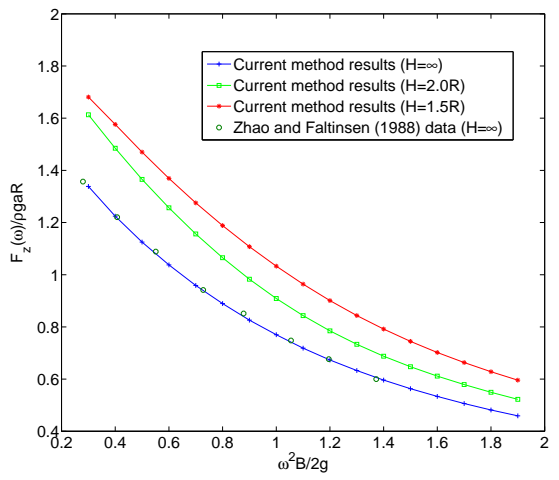

(b) Vertical force $F_{z}(\omega)$

Figure 20: Comparison of horizontal force $F_{x}(\omega)$ (a) and vertical force $F_{z}(\omega)$ (b) determined using the proposed method for water depths $H=\infty, H=2.0 R$ and $H=1.5 R$ and the numerical predictions of Zhao and Faltinsen (1988) for water depth $H=\infty$.

to study the effect of the value of $L$ and the relative position between body and sloping seabed. Numerical simulations were further performed for the circular cylinder experiencing a linear incident wave.

Figure 24 shows comparison of the horizontal force $F_{x}(\omega)$ (a) and vertical force $F_{z}(\omega)$ (b) at water depth $H=2 T$ and a sloping seabed of slope angle $\beta=\arctan 1 / 5$ for $L=2.0 R, L=4.0 R, L=6.0 R$ and $L=8.0 R$ respectively with the cylinder again placed, in each case, at the centre of the sloping seabed. It is observed that an increase of $L$ increases the force responses noticeably in frequency range $0.3<\omega^{2} B / 2 g<1.2$, though this gradually decreases as wave 


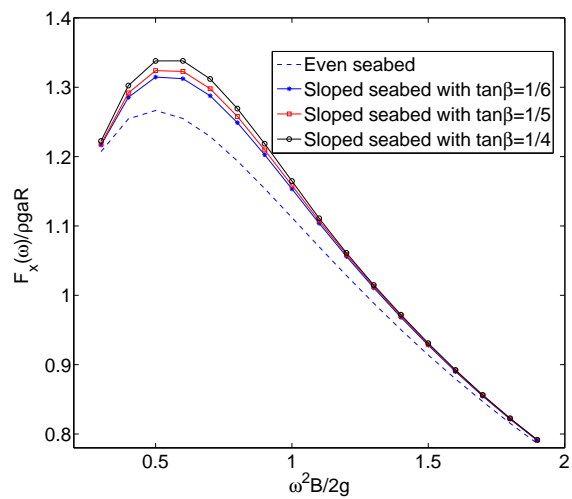

(a) Horizontal force $F_{x}(\omega)$

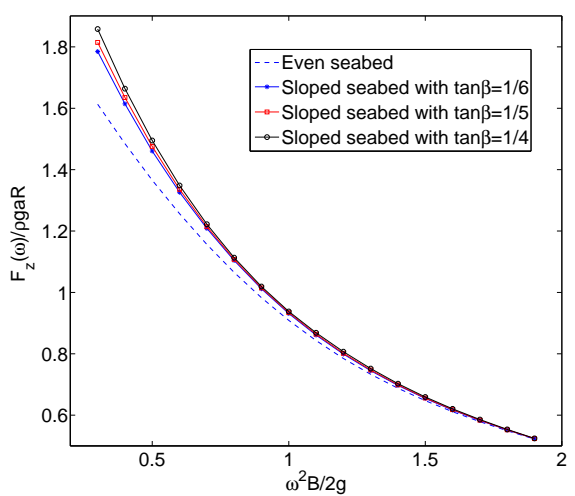

(b) Vertical force $F_{z}(\omega)$

Figure 21: Comparison of horizontal force $F_{x}(\omega)$ (a) and vertical force $F_{z}(\omega)$ (b) for a flat seabed at water depth $H=2 T$ and a sloping seabed of slope angle $\beta=\arctan 1 / 6,1 / 5$ and $1 / 4$ respectively.

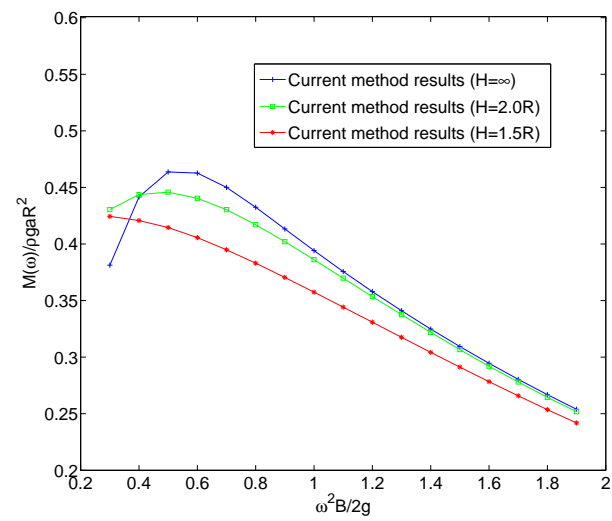

Figure 22: Comparison of moment $M(\omega)$ for water depths $H=\infty, H=2.0 R$ and $H=1.5 R$ respectively.

frequency $\omega^{2} B / 2 g$ increases and the effect is very small when the incident wave frequency $\omega^{2} B / 2 g>1.5$. The larger the base dimension the larger the hydrodynamic response when the incident wave frequency $\omega^{2} B / 2 g<1.5$. It is noticed that for larger values of $L$, the difference between water depth $h_{1}$ and $h_{3}$ are greater, increasing the asymmetry of the configuration and, as seen previously, imposing a more significant influence on the hydrodynamic responses.

To study the relative position or alignment between circular cylinder and sloping seabed and, hence their interaction through hydrodynamic response, the seabed is shifted horizontally through distance $R$ and $2 R$ to both left (negative) 


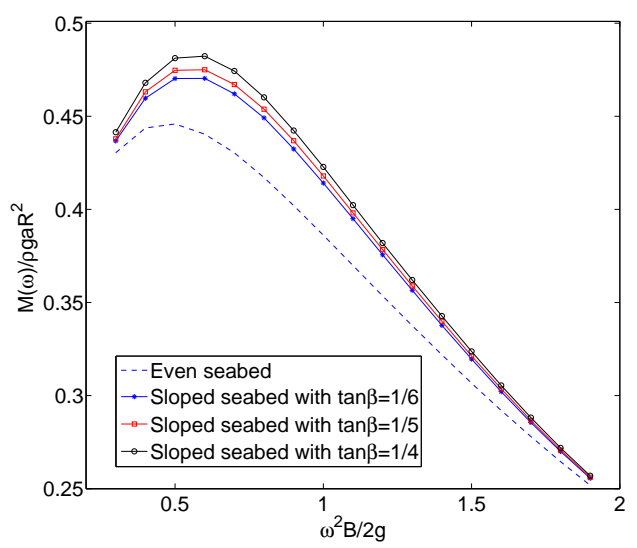

Figure 23: Comparison of moment $M(\omega)$ for a flat seabed at water depth $H=2 T$ and a sloping seabed of slope angle $\beta=\arctan 1 / 6,1 / 5$ and $1 / 4$ respectively.

and right (positive) sides, as shown in Figure 25. Figure 26 shows a comparison of the horizontal force $F_{x}(\omega)$ (a) and vertical force $F_{z}(\omega)$ (b) at water depth $H=2 T$, measured at the centre of the slope, and a sloping seabed of slope angle $\beta=\arctan 1 / 4$ for seabed shifts $-2 R,-R, 0, R$ and $2 R$ respectively. The force responses $F_{x}(\omega)$ and $F_{z}(\omega)$ increase when the seabed shifts to left with reduced separation between cylinder and seabed and they reduce when the seabed shifts to the right. That is, the immediate water depth below the cylinder decreases when the seabed shifts to the left, partially explaining this phenomenon.

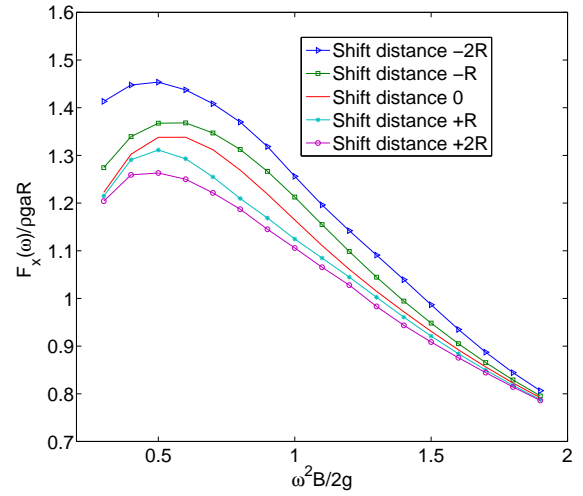

(a) Horizontal force $F_{x}(\omega)$

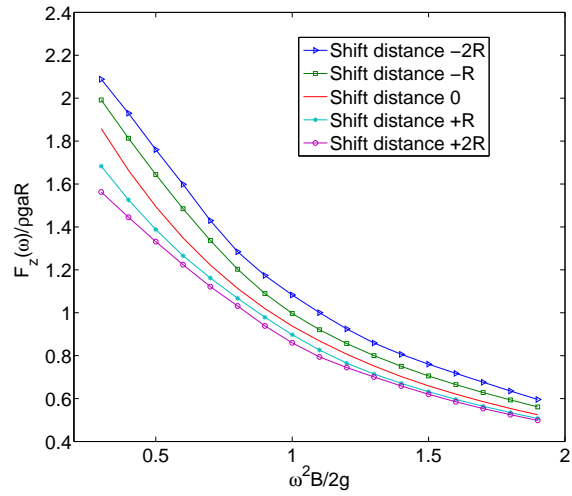

(b) Vertical force $F_{z}(\omega)$

Figure 24: Comparison of horizontal force $F_{x}(\omega)$ (a) and vertical force $F_{z}(\omega)$ (b) for a circular cylinder oscillating above water depth $H=2 T$ and a sloping seabed of slope angle $\beta=$ $\arctan 1 / 5$ for sloping bottom base slope lengths $L=2.0 R, L=4.0 R, L=6.0 R$ and $L=8.0 R$ (see Fig. 3). 


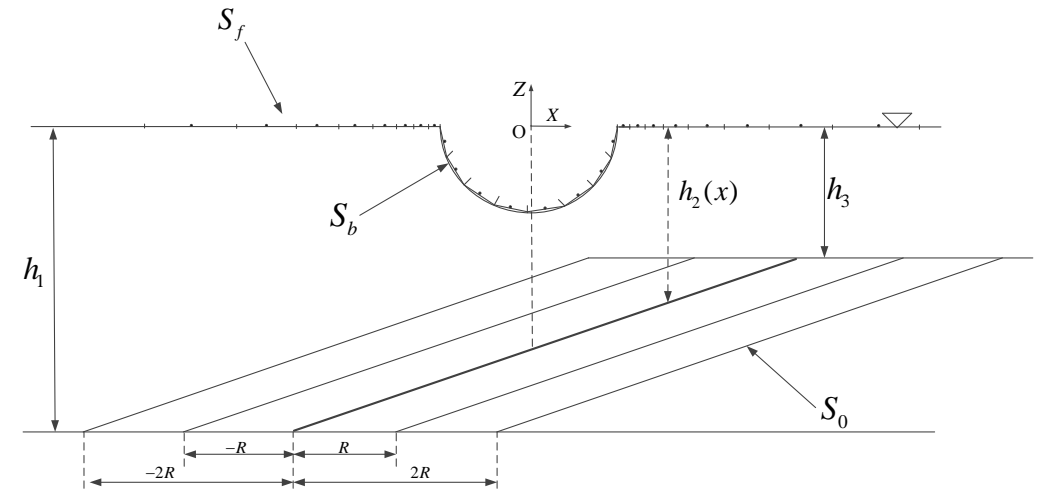

Figure 25: Sketch of seabed shift in horizontal direction to model the relative position and, hence interaction between cylinder and sloping seabed.

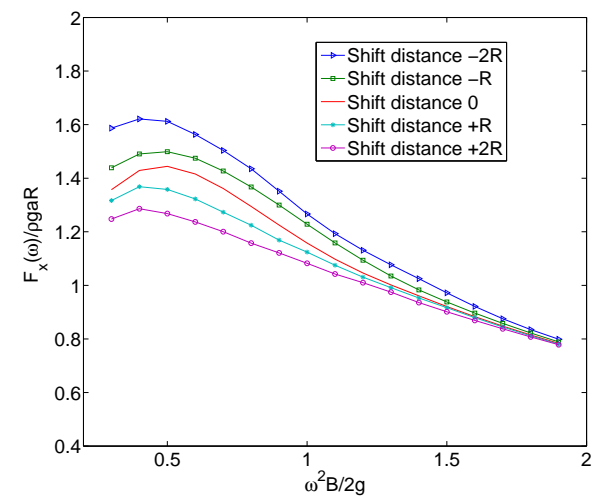

(a) Horizontal force $F_{x}(\omega)$

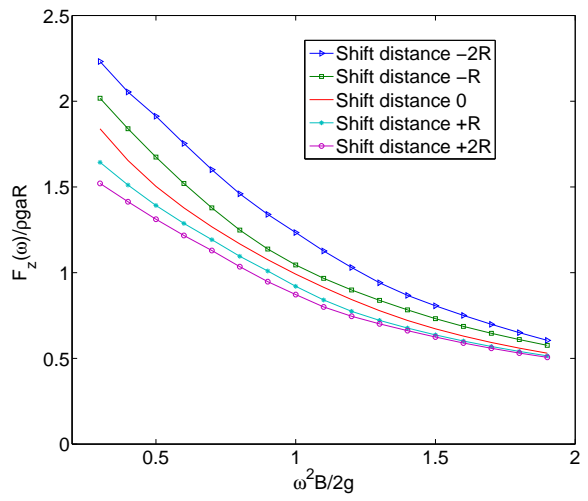

(b) Vertical force $F_{z}(\omega)$

Figure 26: Comparison of predicted horizontal force $F_{x}(\omega)$ (a) and vertical force $F_{z}(\omega)$ (b) associated with a circular oscillating above water depth $H=2 T$, measured at the centre of the slope bottom, and a sloping seabed of slope angle $\beta=\arctan 1 / 4$ for seabed shift $-2 R$, $-R, 0, R$ and $2 R$ as defined in Figure 25 . 


\section{Conclusions}

Two-dimensional wave radiation and diffraction problems associated with topographies relating to a flat or a sloping seabed are investigated by developing a mathematical model adopting a distribution of continuous Rankine source panels. The new seabed Rankine source distribution model is able to deal with asymmetric seabed profiles and a flat seabed. The mathematical model requires only a small additional computational effort compared to an equivalent deep water problem. The general principles underlying the developed seabed source distribution scheme can be easily applied to three-dimensional flat or sloping seabed problems for an acceptable increased level of computational effort.

Wave reflections are incorporated into the mathematical model by satisfying sloping seabed boundary conditions and this approach generates similar results to the introduction of an alternative wave reflection potential. In diffraction problems, when an incident wave moves into a sloping seabed region the phenomenon of wave shoaling occurs and this is also included in the mathematical model.

The mean water depth $H$ influences every hydrodynamic aspect of water wave problems with the exception of vertical and horizontal modes cross coupling hydrodynamic coefficients since their values are zero because of the symmetric nature of the seabed profile. However it is noticed that roll hydrodynamic characteristics are reduced as water depth $H$ decreases. Such behaviour is observed in hydrodynamic coefficients $B_{22}, A_{13} / B_{13}$ and $A_{31} / B_{31}$ in the radiation problem and moment $M$ response in the diffraction problem.

For a sloping seabed environment, in general, the asymmetry existing in this configuration does not influence significantly the diagonal and cross coupling hydrodynamic coefficients. The vertical and horizontal modes cross coupling hydrodynamic coefficients are significantly influenced by the asymmetric nature of the seabed being no longer zero as in the flat seabed case. For example, the values of these hydrodynamic coefficients increase with increasing slope angle $\beta$ or with increasing asymmetry of the seabed topography. The values of these coefficients vary significantly across the frequency range and negative values are observed for all these kinds of hydrodynamic coefficients. The numerical predictions again demonstrate the symmetric characteristics of the hydrodynamic coefficients, $A_{i j}=A_{j i}$ and $B_{i j}=B_{j i}$ regardless of seabed topography.

The force and moment responses are significantly increased by the presence of a sloping seabed. The extent of the sloping surface as defined by the horizontal distance $L$ (see Fig. 25 )and the relative position between sloping seabed and body also play an important role in determining the hydrodynamic responses.

In this investigation, all numerical simulations are constructed adopting a linear model. If nonlinear effects arising from the body and free surface are included, it is expected that the seabed influence will be more pronounced in the hydrodynamic responses, which will form the continuation of this study. 


\section{Acknowledgements}

The authors acknowledge the financial support provided by the Singapore Maritime Institute (SMI) Grant R-261-502-021-592. 


\section{References}

Andersen, P., He, W., 1985. On the calculation of two-dimensional added mass and damping coefficients by simple Green's function technique. Ocean Engineering 12 (5), 425-451.

Athanassoulis, G., Belibassakis, K., 1999. A consistent coupled-mode theory for the propagation of small-amplitude water waves over variable bathymetry regions. Journal of Fluid Mechanics 389, 275-301.

Bai, K., 1977. The added mass of two-dimensional cylinders heaving in water of finite depth. Journal of Fluid Mechanics 81 (01), 85-105.

Bandyk, P., 2009. A body-exact strip theory approach to ship motion computations. Ph.D. thesis, University of Michigan.

Beck, R., Scorpio, S., 1995. A desingularized boundary integral method for fully nonlinear water wave problems. In: Proceedings of the 12th Australasian Fluid Mechanics Conference. pp. 255-258.

Belibassakis, K., 2005. Hydrodynamic analysis of floating bodies in general bathymetry. In: Proceeding of the 24th International Conference on Offshore Mechanics and Arctic Engineering. pp. 449-456.

Belibassakis, K., 2008. A boundary element method for the hydrodynamic analysis of floating bodies in variable bathymetry regions. Engineering Analysis with Boundary Elements 32 (10), 796-810.

Belibassakis, K., Athanassoulis, G., 2004. Three-dimensional Green's function for harmonic water waves over a bottom topography with different depths at infinity. Journal of Fluid Mechanics 510, 267-302.

Buchner, B., 2006. The motions of a ship on a sloped seabed. In: Proceedings of the 25th International Conference on Offshore Mechanics and Arctic Engineering. pp. 339-347.

Cao, Y., Schultz, W., Beck, R., 1991. Three-dimensional desingularized boundary integral methods for potential problems. International Journal for $\mathrm{Nu}-$ merical Methods in Fluids 12 (8), 785-803.

De Hauteclocque, G., Rezende, F., Giorgiutti, Y., Chen, X.-B., 2009. Wave kinematics and seakeeping calculation with varying bathymetry. In: Proceedings of the 28th International Conference on Ocean, Offshore and Arctic Engineering. pp. 515-523.

Feng, A., Bai, W., 2016. Numerical simulation of wave radiation and diffraction problems with current effect. In: Proceedings of the 12th ISOPE Pacific/Asia Offshore Mechanics Symposium. 
Feng, A., Bai, W., You, Y., Chen, Z.-M., Price, W., 2016. A Rankine source method solution of a finite depth, wave-body interaction problem. Journal of Fluids and Structures 62, 14-32.

Feng, A., Chen, Z.-M., Price, W., 2015a. A continuous desingularized source distribution method describing wave-body interactions of a large amplitude oscillatory body. Journal of Offshore Mechanics and Arctic Engineering 137 (2).

Feng, A., Chen, Z.-M., Price, W., 2015b. A desingularized Rankine source method for nonlinear wave-body interaction problems. Ocean Engineering 101, 131-141.

Ferreira, M., Newman, J., 2009. Diffraction effects and ship motions on an artificial seabed. In: Proceedings of the 24th International Workshop on Water Waves and Floating Bodies.

Filippas, E., Belibassakis, K., 2014. Hydrodynamic analysis of flapping-foil thrusters operating beneath the free surface and in waves. Engineering Analysis with Boundary Elements 41, 47-59.

Israeli, M., Orszag, S. A., 1981. Approximation of radiation boundary conditions. Journal of Computational Physics 41 (1), 115-135.

Journée, J., Massie, W., 2001. Offshore Hydrodynamics. First Edition, Delft University of Technology, Section.1-2.

Kim, C., 1969. Hydrodynamic forces and moments for heaving,swaying, and rolling cylinders on water of finite depth. Journal of Ship Research 13, 137164.

Kim, T., Kim, Y., 2013. Numerical analysis on floating-body motion responses in arbitrary bathymetry. Ocean Engineering 62, 123-139.

Kim, Y., Kring, D. C., Sclavounos, P. D., 1997. Linear and nonlinear interactions of surface waves with bodies by a three-dimensional rankine panel method. Applied Ocean Research 19 (5), 235-249.

Koo, W., Kim, M., 2007. Fully nonlinear wave-body interactions with surfacepiercing bodies. Ocean Engineering 34 (7), 1000-1012.

Kring, D., Sclavounos, P., 1995. Numerical stability analysis for time-domain ship motion simulations. Journal of ship research 39 (4), 313-320.

Lamb, H., 1945. Hydrodynamics. 6th edition, Dover Publications, Inc, New York.

Lee, T., 1992. Nonlinear radiation problems for a surface-piercing body. Ph.D. thesis, University of Michigan.

Newman, J. N., 1977. Marine Hydrodynamics. MIT press. 
Sawaragi, T., 1995. Coastal Engineering-Waves, Beaches, Wave-Structure Interactions. Vol. 78. Elsevier, Netherlands.

Tuck, E., 1966. Shallow-water flows past slender bodies. Journal of Fluid Mechanics 26 (01), 81-95.

Tuck, E., 1970. Ship motions in shallow water. Journal of Ship Research 14 (4), $317-328$.

Turkel, E., Yefet, A., 1998. Absorbing pml boundary layers for wave-like equations. Applied Numerical Mathematics 27 (4), 533-557.

Vugts, J., 1968. The hydrodynamic coefficients for swaying, heaving and rolling cylinders in a free surface. International Shipbuilding Progress 15, 251-275.

Vugts, J. H., 1970. The hydrodynamic forces and ship motions in waves. Ph.D. thesis, Delft University of Technology.

Wang, L., Tang, H., Wu, Y., 2015. Simulation of wave-body interaction: A desingularized method coupled with acceleration potential. Journal of Fluids and Structures 52, 37-48.

Wang, L., Tang, H., Wu, Y., 2016. Wave interaction with a surface-piercing body in water of finite depth: a parametric study. Engineering Applications of Computational Fluid Mechanics 10 (1), 512-528.

Williams, A., Lee, H., Huang, Z., 2000. Floating pontoon breakwaters. Ocean Engineering 27 (3), 221-240.

Yeung, R., 1973. A singularity-distribution method for free-surface flow problems with an oscillating body. Ph.D. thesis, University of California, Berkeley.

Yu, Y., Ursell, F., 1961. Surface waves generated by an oscillating circular cylinder on water of finite depth: theory and experiment. Journal of Fluid Mechanics 11 (04), 529-551.

Zhang, X., Bandyk, P., Beck, R., 2010. Time-domain simulations of radiation and diffraction forces. Journal of Ship Research 54 (2), 79-94.

Zhang, X., Beck, R., 2007. Computations for large-amplitude two-dimensional body motions. Journal of Engineering Mathematics 58 (1), 177-189.

Zhao, R., Faltinsen, O. M., 1988. Interaction between waves and current on a two-dimensional body in the free surface. Applied Ocean Research 10 (2), $87-99$. 